\title{
Optimal lattice-structured materials
}

\author{
Mark C. Messner ${ }^{\mathrm{a}, *}$ \\ ${ }^{a}$ Lawrence Livermore National Laboratory, P.O. Box 808, L-227, Livermore, CA 94551, USA
}

\begin{abstract}
This work describes a method for optimizing the mesostructure of lattice-structured materials. These materials are periodic arrays of slender members, resembling efficient, lightweight macroscale structures like bridges and frame buildings. Current additive manufacturing technologies can assemble lattice structures with length scales ranging from nanometers to millimeters. Previous work demonstrates lattice materials have excellent stiffness- and strength-to-weight scaling, outperforming natural materials. However, there are currently no methods for producing optimal mesostructures that consider the full space of possible 3D lattice topologies. The inverse homogenization approach for optimizing the periodic structure of lattice materials requires a parameterized, homogenized material model describing the response of an arbitrary structure. This work develops such a model, starting with a method for describing the long-wavelength, macroscale deformation of an arbitrary lattice. The work combines the homogenized model with a parameterized description of the total design space to generate a parameterized model. Finally, the work describes an optimization method capable of producing optimal mesostructures. Several examples demonstrate the optimization method. One of these examples produces an elastically isotropic, maximally stiff structure, here called the isotruss, that arguably outperforms the anisotropic octet truss topology.
\end{abstract}

Keywords: Lattice materials, microstructures, optimization

\section{Introduction}

Lattice materials apply the design principals of efficient, lightweight macroscale structures, such as buildings and bridges, to mesoscale material architectures. Such lattice-structured materials have excellent stiffness- and strength-to-weight scaling, making these structures efficient for lightweight structural applications [1]. Additive manufacturing technologies can now assemble lattice structures on scales as small as a few nanometers [2] and commercially available technologies can assemble metal lattices with length scales on the order of millimeters [3]. On these scales it becomes reasonable to treat the structures as effective materials with architected lattice mesostructures. Considering lattice materials in this sense neglects the

\footnotetext{
${ }^{*}$ Corresponding author. Tel.: +1-925-423-1540

Email address: messner6@llnl.gov (Mark C. Messner )
} 
fine details of the deformation of the mesostructure in favor of capturing the macroscale, effective response through homogenized modeling.

Viewed as an effective macroscale material architected lattice structures are an excellent framework for material design. Additive manufacturing processes offers a designer control over the printed material mesostructure, which in turn controls the effective material response. The goal of this work is to develop a method for finding optimal mesostructures for structural objectives such as strength and elastic stiffness. Previous methods for optimizing lattice structures focus on a particular unit cell shape, fixing the lattice type, and optimizing the response by adjusting the material distribution inside the cell [4-6]. This work describes a general method for optimizing both the topology of the periodic lattice and the size, position, and number of the connecting struts. This general approach can examine the complete design space of periodic, slender-member lattices and generates novel optimal material structures.

Two general types of methods could be considered for the mesostructural optimization problem. One methodology does not treat the lattice as an effective macroscale material but instead optimizes a unique, non-periodic strut-and-joint type structure over a whole spatial domain. Such structures are not strictly lattice materials, as they are not periodic. However, fundamentally these structures apply the same design principals as lattice-structured materials. Previous methods for finding such structures often apply discrete truss/frame optimization methods [7,8] originating from the ground structure method of Dorn et al. [9]. Bendsoe et al [10] provide an early review on these methods. However, similar slender-member structures also arise from continuum optimization methods, for example see Rozvany et al. [11], Olhoff et al. [12], and many others.

An alternate approach involves a two scale design methodology. At each material point the optimizer calls a parameterized, homogenized model to determine the local, effective material properties. These methods embed this homogenized response into a macroscale structural optimization problem with the goal of finding the optimal local mesostructure, as described by the parameters of the homogenized material model, potentially at each point in a spatial domain (see [13-15]). Such methods have been applied to static linear elastic, two dimensional lattices by enforcing periodicity constraints on the mesoscale problems [16, 17]. Le et al. [18] applied a similar optimization methodology to a different mesostructure - sequentially ranked laminar composites - to channel elastodynamic energy through a composite structure. Le et al. and related work on rank laminar composites do not enforce microstructure compatibility - local microstructures at adjacent material points may be completely dissimilar. This means the optimized structures may not be feasible to manufacture. The approach described here defines the optimization problem over an intrinsicallyperiodic design space. The resulting optimal mesostructures are then inherently compatible and feasible to manufacture. 
A notable intermediate between these two types of methods is that of Sigmund [19] and later related work by Sigmund and colleagues (e.g. [20]) as well as Liu et al. [21], andHuang et al. [22]. These methods design a single unit cell using a selected optimization technique - the ground structure method in the case of Sigmund. The technique then computes the effective, homogenized properties of a structure consisting of a tiled array of these discrete unit cells. These methods come closest to the approach described here. However, these previous approaches assume some a priori unit cell symmetry, often cubic or square - though Diaz [23] investigated other cell types in $2 \mathrm{D}$. Therefore, they do not simultaneously examine the complete design space of all lattice materials. The method described here overcomes this limitation to examine a complete subspace of the lattice design space, up to a selected limit on the lattice complexity described in Section 2.

This work optimizes an effective lattice material structure through homogenized models of periodic mesostructures. Such a framework for optimizing lattice mesoarchitectures requires two subcomponents. One subcomponent is a homogenized material model that converts a periodic, strut-and-joint material, described by a primitive unit cell, to its effective, macroscale properties. The model described here builds on previous models by Hutchinson and Fleck [24], Phani et al. [25], and the author [26] and represents the long-wavelength linear elastic properties and approximate failure surface of general, three dimensional lattice materials - both stretch and bend dominated. The form of the model, developed in Section 2, ensures that the optimal structures will remain collections of slender members connected at joints. The second necessary subcomponent is a parameterized description of the lattice design space. This parameterization, developed in Section 3, describes any possible lattice topology as a compact vector of real numbers. Using this description an optimizer can explore the entire design space to find optimal mesostructures. The form of the parameterization ensures the mesostructures have the required translational symmetry without any constraints on the optimization problem itself. Finally, an optimization method can work with the parameterized model, combining the parameterized description of the space of lattice materials with the general model for the macroscale response, to find optimal structures. This work applies the optimization method to describe two novel mesostructures.

The optimization problems addressed in this work are inherently mesostructural. The optimizations aim to find generically useful mesostructures, tiled periodically over a large domain, for a given structural objective like stiffness or strength. However, the concepts developed here could be extended to solve macrostructural optimization problems by finding the optimal mesostructure at each point in a spatial optimization domain. The final section of this work briefly describes such an extension of the method, including a discussion of additional compatibility constraints required to produce manufacturable materials. The discussion also describes the formation of elastic boundary layers in the optimized periodic structures and how those boundary layers can affect the properties of the effective material. Finally, Section 5 summarizes the 


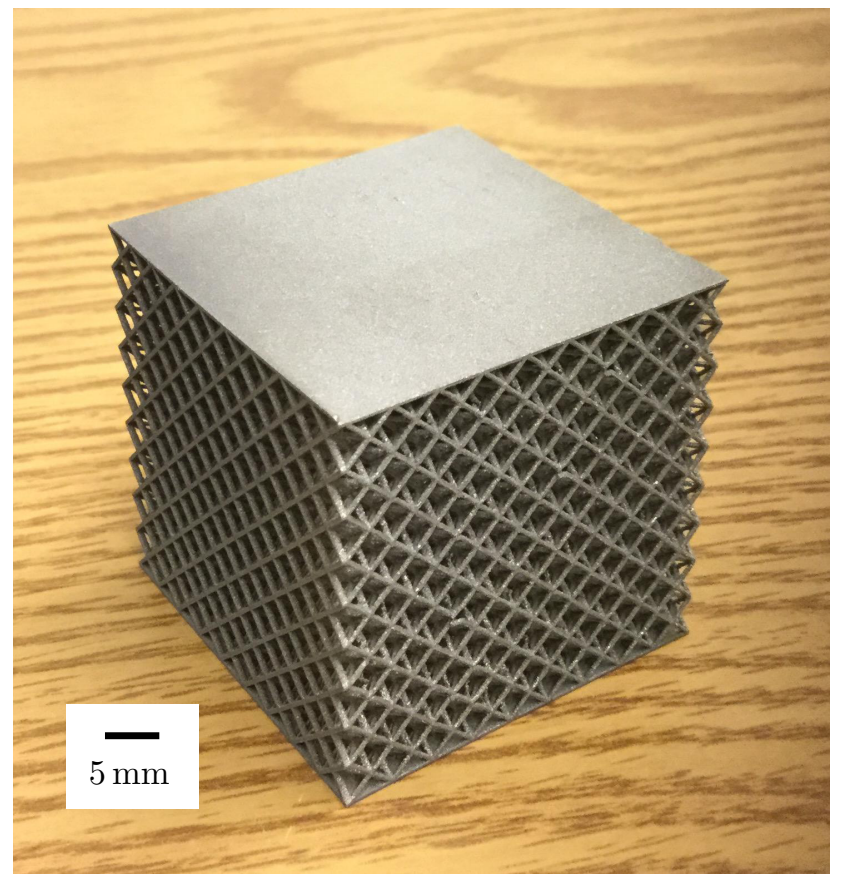

Figure 1: An example of a lattice structure constructed by a selective laser melting process. The structure is a $9 \mathrm{x} 9 \mathrm{x} 9$ tiling of the octet truss unit cell topology [27]. The bulk material is Ti-6Al-4V and the cell geometry produces an effective material with $10 \%$ relative density.

conclusions developed in this work.

\section{Linear elastic and limit state analysis of general lattice materials}

This work considers the response of periodic lattice structures of the kind shown in Fig. 1, described as collections of slender members called struts connected at node locations called joints.

The joints are arranged periodically, such that if a joint is located at position $\mathbf{x}_{0}^{(k)}$ then a joint $i$ is also located at

$$
\mathbf{x}_{i}^{(k)}=\mathbf{x}_{0}^{(k)}+\sum_{j=1}^{n} n_{j}^{(i)} \mathbf{a}_{j}
$$

for all integers $n_{j}^{(i)}$, where $\mathbf{a}_{i}$ are the lattice vectors and $n$ is the lattice dimensionality $-n=2$ for $2 \mathrm{D}$ and $n=3$ for 3D. This description divides joints into equivalent sets $k$ based on their offset $\mathbf{x}_{0}^{(k)}$ so that

$$
J_{k}=\left\{\mathbf{x}_{i} \mid \mathbf{x}_{i}=\mathbf{x}_{0}^{(k)}+\sum_{j=1}^{n} n_{j}^{(i)} \mathbf{a}_{j}\right\}
$$

describes a joint set, for all integers $n_{i}$. The coordinate system can be selected so that one joint set has $\mathbf{x}_{0}=0$. Call this set $J_{0}$. If the lattice joints are all contained in this single set call the lattice simple. If 
multiple joint sets are required to describe the structure call the lattice complex.

A collection of joint basis vectors can equivalently describe the periodic joint positions. With this description, the equation

$$
\mathbf{x}_{i}^{(k)}=\sum_{j=1}^{n} n_{j}^{(i)} \mathbf{a}_{j}+\mathbf{j}^{(k)}
$$

describes all joint positions for integer multipliers $n_{j}^{(i)}$, with $\mathbf{j}^{(k)} \in B$, a set of vectors. Setting the origin at a joint position, as before, a simple lattice has $B=\{\mathbf{0}\}$ and the displacement vectors from the previous joint set description are the joint basis vectors: $\mathbf{j}^{(k)}=\mathbf{x}_{0}^{(k)}$. Let the degree of the lattice be given by $d=|B|-1$, so that $d=0$ for a simple lattice.

Consider the primitive unit cell, defined as the volume of space enclosed by the parallelogram (2D) or parallelepiped (3D) determined by the lattice vectors $\mathbf{a}_{i}$. The points described by the joint basis vectors, offset from $\mathbf{0}$, all fall inside this primitive cell and can be expressed as fractional lattice coordinates, so that

$$
\mathbf{j}^{(k)}=\sum_{j=1}^{n} m_{j} \mathbf{a}_{j}
$$

with $m_{i} \in[0,1]$. The primitive unit cell encompasses several equivalent joints in the set $J_{0}-4$ in $2 \mathrm{D}$ or 8 in 3D - but only one joint in each of the remaining joint sets.

The primitive cell of the lattice describes the entire structure under translational symmetry given by the lattice vectors. This work assumes the elastic response of this unit cell can be described by a homogeneous, undamped linear model, parameterized by the position and rotation of each joint in the cell as a function of time. Concatenating these joint degrees of freedom into a vector $\mathbf{u}$, the model takes the form

$$
\mathbf{M u}+\mathbf{K u}=\mathbf{0}
$$

where $\ddot{\mathbf{u}}=\frac{d^{2}}{d t^{2}} \mathbf{u}, \mathbf{M}$ is the unit cell mass matrix, and $\mathbf{K}$ is the unit cell stiffness matrix. The subsequent derivation is agnostic to the particular structural theory used to generate this linear model, though the theory should adequately describe the deformation mechanisms occurring in the structure. A later section describes the particular structural theory used for example problems in this work: a frame element considering axial deformation, torsion, and Euler-Bernoulli bending in two directions.

\subsection{Bloch wave solution}

Given the preceding description of the problem a superposition of Bloch waves describes the exact solution for the dynamic deformation of the periodic lattice. The Bloch theory describes a propagating wave with wavevector $\mathbf{k}$ and frequency $\omega$ as the superposition of a displacement field periodic over the lattice and a 
spatial phase shift. For each joint $i$ in joint set $k$ the lattice joint displacements and rotations at all the lattice-equivalent points $\mathbf{x}_{i}^{(k)}$ are given by:

$$
\mathbf{u}_{i}^{(k)}=\check{\mathbf{u}}^{(k)} e^{i\left(\mathbf{k} \cdot \mathbf{x}_{i}^{(k)}-\omega t\right)}
$$

Here $\check{\mathbf{u}}^{(k)}$ represents the periodic part of the displacement field and the remainder of the expression describes the spatial phase shift in terms of the wavevector $\mathbf{k}$ and the joint position $\mathbf{x}_{i}^{(k)}$. Because each joint set $k$ shares the same $\check{\mathbf{u}}^{(k)}$ this part of the displacement is periodic over the lattice. Finding the complete Bloch solution for a given wavector $\mathrm{k}$ requires finding the corresponding collection of modal frequencies $\omega$ and nodal mode shapes $\check{\mathbf{u}}^{(k)}$.

Depending on the topology of the lattice a Bloch wave with wavevector $\mathbf{k}$ can propagate in several deformation modes. These modes are the solutions to an eigenvalue problem formulated by applying the Bloch phase shift condition to the unit cell linear model (Eq. 5). Divide joints in the primitive unit cell into two types: those in $J_{0}$ and those in the remaining joint sets $J_{k}$. Equation 6 ties together the displacements and rotations for joints in $J_{0}$. The remaining degrees of freedom, belonging to the joint sets $J_{1}, J_{2}, \ldots$ are free, because only one joint of each type appears in the unit cell. Similar to [25] and the author's previous work [26], these Bloch wave constraints can be restated as transformations on the unit cell mass and stiffness matrices, so that the new statement of equilibrium is

$$
\begin{array}{r}
\mathbf{T}(\mathbf{k})^{H} \mathbf{M T}(\mathbf{k}) \ddot{\mathbf{u}}+\mathbf{T}(\mathbf{k})^{H} \mathbf{K} \mathbf{T}(\mathbf{k}) \hat{\mathbf{u}}=\mathbf{0} \\
\hat{\mathbf{M}}(\mathbf{k}) \ddot{\hat{\mathbf{u}}}+\hat{\mathbf{K}}(\mathbf{k}) \hat{\mathbf{u}}=\mathbf{0}
\end{array}
$$

with $\hat{\mathbf{u}}=\check{\mathbf{u}} e^{-i \omega t}$. The entries of the transformation matrix impose the Bloch constraints on the tied degrees of freedom while leaving the free degrees of freedom untransformed. Carrying out the time derivatives in Eq. 7 results in the generalized eigenvalue problem

$$
\hat{\mathbf{K}}(\mathbf{k}) \check{\mathbf{u}}=\omega^{2} \hat{\mathbf{M}}(\mathbf{k}) \check{\mathbf{u}} .
$$

Given the wavevector $\mathbf{k}$, solving this equation gives the modal frequencies $\omega$ and the corresponding mode shapes, described by $\tilde{\mathbf{u}}$.

The results of solving Eq. 8 over wavevector space are often summarized in dispersion relations, as shown in Fig. 2. These plots illustrate the relation between wavenumber and frequency for all modes in the periodic structure for a stretch dominated lattice, the octet truss (Fig. 2a), and a bend dominated lattice, the rhombic 
dodecahedron (Fig. 2b). This plot shows the relation for wave propagation in the direction indicated by the diagrams at the bottom of the figure. The plots then only show one line through the irreducible Brillouin zone, starting at the origin, in order highlight the correspondence of the long wavelength theory - developed in the next section - with the Bloch response at low frequencies. Because both of these structures have cubic symmetry the range $k \in[0, \pi / a]$ contains the unique dispersion relation, with $a$ the lattice parameter.

\subsection{Elastic properties in the long wavelength limit}

Consider the long wavelength limit of the Bloch solution described by Eq. 6, letting the wavevector $\mathbf{k} \rightarrow \mathbf{0}$. This limit describes the propagation of waves with long wavelengths and low frequencies or, equivalently, deformation modes that coordinate over many unit cells. The author's previous work [26] demonstrates that, for typical lattice structures, approximating the dynamics of the material with the long wavelength limit preserves accuracy for low frequency vibration and quasi-static deformation.

In the long wavelength limit the Bloch phase shift vanishes. Furthermore, the Cauchy-Born hypothesis sets the fixed, $J_{0}$ joints translational displacements affine with the imposed, macroscale strain

$$
\mathbf{d}_{i}^{(0)}=\boldsymbol{\varepsilon} \mathbf{x}_{i}^{(0)}
$$

The $J_{0}$ rotations $-\boldsymbol{\theta}_{i}^{(0)}$ - are tied together to enforce periodicity but they are not related to any macroscale measure of deformation. The remaining degrees of freedom, associated with the remaining joint sets, are unconstrained. The transformation matrix $\tilde{\mathbf{T}}$ ties the $J_{0}$ rotational degrees of freedom together and transforms the unit cell linear model to

$$
\begin{aligned}
\tilde{\mathbf{T}}^{T} \mathbf{M} \tilde{\mathbf{T}} \ddot{\tilde{\mathbf{u}}}+\tilde{\mathbf{T}}^{T} \mathbf{K} \tilde{\mathbf{T}} \tilde{\mathbf{u}} & =\mathbf{0} \\
\tilde{\mathbf{M}} \ddot{\tilde{\mathbf{u}}}+\tilde{\mathbf{K}} \tilde{\mathbf{u}} & =\mathbf{0} .
\end{aligned}
$$

Then partition the reduced set of degrees of freedom $\tilde{\mathbf{u}}$ so that, in $3 \mathrm{D}$ :

$$
\tilde{\mathbf{u}}=\left[\begin{array}{lllllllllll}
\mathbf{d}_{1}^{(0)} & \mathbf{d}_{2}^{(0)} & \cdots & \mathbf{d}_{8}^{(0)} & \mid & \boldsymbol{\theta}^{(0)} & \mathbf{d}^{(1)} & \boldsymbol{\theta}^{(1)} & \cdots & \mathbf{d}^{(d)} & \boldsymbol{\theta}^{(d)}
\end{array}\right]=\left[\begin{array}{lll}
\tilde{\mathbf{u}}_{x} & \mid & \tilde{\mathbf{u}}_{f}
\end{array}\right]
$$

In this partitioning $\mathbf{d}_{i}^{(0)}$ are the eight displacements associated with the joints in $J_{0}$ - affine to the macroscale strain, according to the Cauchy-Born hypothesis $-\boldsymbol{\theta}^{(0)}$ are the master $J_{0}$ rotations and the $\mathbf{d}^{(k)} / \boldsymbol{\theta}^{(k)}$ pairs are the degrees of freedom for the remaining joint sets. The global partition $\mathbf{u}_{x} / \mathbf{u}_{f}$ lumps the degrees of freedom into a group fixed to the macroscale strain - the displacements $\mathbf{d}_{0}^{(i)}$ - and a group not tied to any macroscale measure of deformation - the master $J_{0}$ rotation and the remaining free degrees of freedom. 
Based on this partition divide the transformed unit cell stiffness and mass matrices into blocks:

$$
\tilde{\mathbf{K}}=\left[\begin{array}{cc}
\tilde{\mathbf{K}}_{x x} & \tilde{\mathbf{K}}_{x f} \\
\tilde{\mathbf{K}}_{f x} & \tilde{\mathbf{K}}_{f f}
\end{array}\right]
$$

and

$$
\tilde{\mathbf{M}}=\left[\begin{array}{cc}
\tilde{\mathbf{M}}_{x x} & \tilde{\mathbf{M}}_{x f} \\
\tilde{\mathbf{M}}_{f x} & \tilde{\mathbf{M}}_{f f}
\end{array}\right]
$$

Note, by the symmetry of the mesoscale unit cell stiffness matrices, that $\tilde{\mathbf{K}}_{x f}=\tilde{\mathbf{K}}_{f x}^{T}$ and similarly for the mass matrix. This partitioning follows that of Gong et al. [28].

From this decomposition write the strain energy in a single unit cell as

$$
W=\frac{1}{2} \tilde{\mathbf{u}}^{T} \tilde{\mathbf{K}} \tilde{\mathbf{u}}=\frac{1}{2}\left[\tilde{\mathbf{u}}_{x}^{T} \tilde{\mathbf{K}}_{x x} \tilde{\mathbf{u}}_{x}+\tilde{\mathbf{u}}_{x}^{T} \tilde{\mathbf{K}}_{x f} \tilde{\mathbf{u}}_{f}+\tilde{\mathbf{u}}_{f}^{T} \tilde{\mathbf{K}}_{f x} \tilde{\mathbf{u}}_{x}+\tilde{\mathbf{u}}_{f}^{T} \tilde{\mathbf{K}}_{f f} \tilde{\mathbf{u}}_{f}\right]
$$

Use condensation to solve for the free degrees of freedom, i.e. $\tilde{\mathbf{u}}_{f}=-\tilde{\mathbf{K}}_{f f}^{-1} \cdot \tilde{\mathbf{K}}_{f x} \cdot \tilde{\mathbf{u}}_{x}$. Substituting into Eq. 14 yields

$$
W=\frac{1}{2} \tilde{\mathbf{u}}_{x}^{T}\left[\tilde{\mathbf{K}}_{x x}-\tilde{\mathbf{K}}_{x f} \tilde{\mathbf{K}}_{f f}^{-1} \tilde{\mathbf{K}}_{f x}\right] \tilde{\mathbf{u}}_{x}
$$

Because this solution is periodic, the strain energy density in the lattice material is

$$
\bar{\varphi}=\frac{1}{2 V} \tilde{\mathbf{u}}_{x}^{T}\left[\tilde{\mathbf{K}}_{x x}-\tilde{\mathbf{K}}_{x f} \tilde{\mathbf{K}}_{f f}^{-1} \tilde{\mathbf{K}}_{f x}\right] \tilde{\mathbf{u}}_{x}
$$

with $V$ the volume of the primitive unit cell $-V=\left|\mathbf{a}_{1} \cdot\left(\mathbf{a}_{2} \times \mathbf{a}_{3}\right)\right|$ in 3D. Let the final, reduced stiffness be

$$
\overline{\mathbf{K}}=\tilde{\mathbf{K}}_{x x}-\tilde{\mathbf{K}}_{x f} \tilde{\mathbf{K}}_{f f}^{-1} \tilde{\mathbf{K}}_{f x}
$$

Finally, the linear relation

$$
\left(\tilde{\mathbf{u}}_{x}\right)_{i}=E_{i j k} \varepsilon_{j k}
$$

relates the macroscale strain to the microscale, fixed displacements through the Cauchy-Born hypothesis. $E_{i j k}$ is blocked so that for fixed joint $f$

$$
E_{i j k}^{(f)}=\delta_{i j} x_{k}^{(f)}
$$

Substituting Eqs. 17 and 18 into Eq. 16 simplifies to

$$
\bar{\varphi}=\frac{1}{2 V} \varepsilon: \mathbf{E}^{T} \cdot\left[\overline{\mathbf{K}}_{x x}-\overline{\mathbf{K}}_{x f} \overline{\mathbf{K}}_{f f}^{-1} \overline{\mathbf{K}}_{f x}\right] \cdot \mathbf{E}: \boldsymbol{\varepsilon}
$$


which expresses the strain energy density in the homogenized material as a quadratic functional of strain. Here the transpose on the third-order tensor $\mathbf{E}$ reverses the indices so that $E_{i j k}$ transforms to $E_{k j i}$, the dot operator $\cdot$ represents single contraction over adjacent indices in the Einstein convention $\left(\mathbf{M} \cdot \mathbf{X}=M_{i j} X_{j k l}\right)$, and the double dot operator : represents double contraction over adjacent indices $\left(\mathbf{M}: \mathbf{X}=M_{i j} X_{i j k}\right)$. Taking the second derivative with respect to the strain produces the homogenized effective elasticity tensor

$$
\overline{\mathbf{C}}=\frac{1}{V} \mathbf{E}^{T} \cdot\left[\overline{\mathbf{K}}_{x x}-\overline{\mathbf{K}}_{x f} \overline{\mathbf{K}}_{f f}^{-1} \overline{\mathbf{K}}_{f x}\right] \cdot \mathbf{E}
$$

The author's previous work illustrates the importance of microinertial effects in periodic lattice materials [26]. These microinertial effects represent the anisotropic distribution of inertial force in the mesoscale lattice. In the long wavelength limit microinertial effects can be expressed as an effective density matrix $\mathbf{P}$ describing the inertial body force in the homogenized, macroscale material as

$$
\mathbf{f}=\mathbf{P a}
$$

with $\mathbf{f}$ the inertial body force, $\mathbf{P}$ the effective density matrix, and a the macroscale acceleration field. The effective density matrix can be computed from the kinetic energy density in the homogenized continuum. Starting from the decomposition of the mass matrix into fixed and free blocks the kinetic energy density is

$$
\bar{\psi}=\frac{1}{2 V} \dot{\tilde{\mathbf{u}}}^{T} \tilde{\mathbf{M}} \dot{\tilde{\mathbf{u}}}=\frac{1}{2}\left[\dot{\tilde{\mathbf{u}}}_{x}^{T} \tilde{\mathbf{M}}_{x x} \dot{\tilde{\mathbf{u}}}_{x}+\dot{\tilde{\mathbf{u}}}_{x}^{T} \tilde{\mathbf{M}}_{x f} \dot{\tilde{\mathbf{u}}}_{f}+\dot{\tilde{\mathbf{u}}}_{f}^{T} \tilde{\mathbf{M}}_{f x} \dot{\tilde{\mathbf{u}}}_{x}+\dot{\tilde{\mathbf{u}}}_{f}^{T} \tilde{\mathbf{M}}_{f f} \dot{\tilde{\mathbf{u}}}_{f}\right]
$$

Assuming small deformations the unit cell mass and stiffness matrices are constant in time. Therefore, by condensation $\dot{\tilde{\mathbf{u}}}_{f}=-\tilde{\mathbf{K}}_{f f}^{-1} \tilde{\mathbf{K}}_{f x} \dot{\tilde{\mathbf{u}}}_{x}$. Substituting this expression into Eq. 23 and simplifying produces

$$
\bar{\psi}=\frac{1}{2 V} \dot{\tilde{\mathbf{u}}}_{x}^{T}\left[\tilde{\mathbf{K}}_{x f} \tilde{\mathbf{K}}_{f f}^{-1} \tilde{\mathbf{M}}_{f f} \tilde{\mathbf{K}}_{f f}^{-1} \tilde{\mathbf{K}}_{f x}-\tilde{\mathbf{K}}_{x f} \tilde{\mathbf{K}}_{f f}^{-1} \cdot \tilde{\mathbf{M}}_{f x}-\tilde{\mathbf{M}}_{x f} \tilde{\mathbf{K}}_{f f}^{-1} \tilde{\mathbf{K}}_{f x}+\tilde{\mathbf{M}}_{x x}\right] \dot{\tilde{\mathbf{u}}}_{x}
$$

The let reduced mass matrix be

$$
\overline{\mathbf{M}}=\tilde{\mathbf{K}}_{x f} \tilde{\mathbf{K}}_{f f}^{-1} \tilde{\mathbf{M}}_{f f} \tilde{\mathbf{K}}_{f f}^{-1} \tilde{\mathbf{K}}_{f x}-\tilde{\mathbf{K}}_{x f} \tilde{\mathbf{K}}_{f f}^{-1} \tilde{\mathbf{M}}_{f x}-\tilde{\mathbf{M}}_{x f} \tilde{\mathbf{K}}_{f f}^{-1} \tilde{\mathbf{K}}_{f x}+\tilde{\mathbf{M}}_{x x}
$$

so that

$$
\bar{\psi}=\frac{1}{2 V} \dot{\tilde{\mathbf{u}}}_{x}^{T} \overline{\mathbf{M}} \dot{\tilde{\mathbf{u}}}_{x}
$$


Finally, assume that the microscale velocity field matches the macroscale field. That is, let

$$
\mathbf{v}=\mathbf{G} \dot{\tilde{\mathbf{u}}}_{x}
$$

with blocks of the tensor $\mathbf{G}$ for each fixed joint $f$

$$
G_{i k}^{(f)}=\delta_{i k}
$$

matching the velocity of the $J_{0}$ joints on the microscale to the macroscale velocity v. Substituting Eqs. 25 and 27 into Eq. 24 generates a functional for the kinetic energy density, quadratic in terms of the macroscale velocity

$$
\bar{\psi}=\frac{1}{2 V} \mathbf{v G}^{T} \overline{\mathbf{M}} \mathbf{G} \mathbf{v}
$$

Finally, taking two derivatives with respect to the velocity yields the effective density matrix

$$
\overline{\mathbf{P}}=\frac{1}{V} \cdot \mathbf{G}^{T} \overline{\mathbf{M}} \mathbf{G}
$$

Equations 21 and 30 take the discrete, microscale unit cell model to a continuous, macroscale, homogenized model representing the deformation of a large collection of translationally symmetric cells. Given the linear model representing the response of the unit cell computing the macroscale properties is a straightforward operation on blocks of the transformed discrete mass and stiffness matrices. Figure 2 shows the dispersion relation generated by this procedure for two different microscale topologies.

This procedure linearizes the Bloch dispersion relation about $\mathbf{k}=\mathbf{0}$. As such, the homogenization is only accurate for low frequency vibration and does not accurately represent higher frequency phenomenon like band gaps and veering. Other research focuses on homogenization in the opposite limit - high frequency vibration [29-31]. These theories could also be used to optimize periodic structures. The choice of theory should depend on the conditions the lattice will see in actual use.

\subsection{Limit states}

Designing a lattice material for strength requires some measure of the load carrying capacity of an arbitrary periodic structure. The slender struts in a metal lattice material fail through the interaction of plastic deformation and geometric instability. The locus of stress states causing failure in at least one strut is a reasonable measure of the strength of an infinite, periodic material. By analogy to yield surfaces in conventional plasticity models call the surface describing this locus of points in stress space the failure surface. The zero isosurface of the failure function $f(\boldsymbol{\sigma})$ describes the surface. For a given stress state $\boldsymbol{\sigma}$, 
$f(\boldsymbol{\sigma})>0$ indicates at least one strut has failed. Note this function only describes the onset of localization and does not represent any potential post-buckled capacity. Depending on the lattice topology the structure may have considerable post-buckled strength, particularly in the compaction regime where struts begin to come into contact [32].

Two factors complicate the evaluation of the failure function: 1) interaction between plastic bending and buckling and 2) local buckling modes. Strain is not constant over a section undergoing plastic bending making it a difficult stress state to represent with 1D elements. However, a model must resolve this nonuniform plastic deformation in order to assess the interaction of bending moment and axial force leading to buckling or section yielding. Furthermore, there are two kinds of buckling modes to consider in lattice structures: long-wavelength buckling where the structure fails periodically - the same member(s) in each unit cell fail - and local buckling modes coordinating over a finite number of cells.

Gong et al. [28] describe a procedure for calculating the local buckling strength of a lattice. Their description was specific to a particular cell topology and only considers geometric nonlinearity but the procedure could be extended to other unit cells and material nonlinearity. The method starts with an incremental nonlinear analysis of a unit cell in the long-wavelength limit. The unit cell analysis must consider both geometric and material nonlinearities in order to capture the interaction between buckling and yielding. At each increment of load, the method calculates the long wavelength, periodic deformation of the structure. The method then evaluates the discrete unit cell stiffness matrix in the deformed configuration. Next, the procedure calculates a Bloch wave shifted stiffness matrix - analogous to Eq. 7 - accounting for the local buckling modes. This shift is a function of wavevector and the shifted stiffness can be described as $\mathbf{K}_{\text {shift }}(\mathbf{k})$. If the shifted stiffness matrix has $\operatorname{det} \mathbf{K}_{\text {shift }}(\mathbf{k})=0$ for any wavevector $\mathbf{k}$ then a buckling mode exists at that deformation state with a mode shape describe by the Bloch wavevector $\mathbf{k}$. In practice it is sufficient to consider wavevectors in the first Brillouin zone. This procedure must be repeated across stress space in order to describe the failure surface.

This process is computationally expensive, involving both incremental nonlinear analysis of the unit cell, starting from zero load the extending throughout stress space, and the search for potential buckling modes over the first Brillouin zone at each load increment. For the sake of efficient evaluation of the model inside an optimization loop this work adopts a simplified approach requiring two approximations. The first approximation is again taking the long wavelength limit, essentially setting $\mathbf{k}=\mathbf{0}$ in the approach of Gong et al. [28]. This means the model will not consider local buckling modes, only global, periodic buckling. The second approximation eschews nonlinear analysis of the unit cell in favor of a linear analysis coupled with interaction equations approximately describing the interaction of plasticity and geometric instability.

Given the unit cell strut forces as a function of stress, consider a collection of limit state functions of the 
form $l_{j}\left(\mathbf{f}_{i}, \mathbf{p}_{i}\right)$ where $\mathbf{f}_{i}$ are the forces in strut $i, \mathbf{p}_{i}$ is a general set of strut parameters, such as length, cross sectional area, and moments of inertia, and $l_{j}$ is a scalar with the interpretation of $l_{j} \leq 0$ indicates a strut has not failed and $l_{j}>0$ indicates a failed strut. These limit state functions approximately describe strut failure mechanisms, such as buckling and yielding, and the interaction between multiple failure modes. A later section describes a particular limit state function based on the interaction equations in the AISC Steel Construction Manual [33]. Given a function relating the stress state of the material to the periodic, long wavelength strut forces

$$
f(\boldsymbol{\sigma})=\max _{i} \max _{j} l_{j}\left(\mathbf{f}_{i}(\boldsymbol{\sigma}), \mathbf{p}_{i}\right)
$$

describes the failure function, where the outer maximization $(i)$ looks at all the struts in the periodic unit cell and the inner maximization $(j)$ considers each limit state function in the collection. In the long wavelength limit the forces in the struts are periodic across the material. Therefore, a model only needs to consider a single unit cell to determine the failure function for the entire periodic structure.

The remaining challenge is evaluating the function $\mathbf{f}_{i}(\boldsymbol{\sigma})$ describing the forces in each periodic strut for stress state $\boldsymbol{\sigma}$. The model can reuse some of the analysis performed to evaluate the long wavelength elastic properties of the effective material. Starting with the homogenized effective elastic stiffness of the material, the long wavelength strain state in the structure is

$$
\varepsilon=\overline{\mathbf{C}}^{-1}: \sigma
$$

Given the strains, the Cauchy-Born tensor $\mathbf{E}$ takes the strain state to the fixed joint degrees-of-freedom:

$$
\tilde{\mathbf{u}}_{x}=\mathbf{E}: \varepsilon
$$

Define the matrix $\mathbf{D}_{x f}$ that takes the fixed degrees of freedom to the tied-rotation generalized displacement vector $\tilde{\mathbf{u}}$ :

$$
\tilde{\mathbf{u}}=\mathbf{D}_{x f} \tilde{\mathbf{u}}_{x}
$$

with $\mathbf{D}_{x f}$ the blocked matrix

$$
\mathbf{D}_{x f}=\left[\begin{array}{c}
\mathbf{I} \\
-\tilde{\mathbf{K}}_{f f}^{-1} \tilde{\mathbf{K}}_{f x}
\end{array}\right]
$$

Then the transformation matrix $\tilde{\mathbf{T}}$ carries the tied-rotation displacement vector $\tilde{\mathbf{u}}_{x}$ to the full generalized displacement vector $\mathbf{u}$ :

$$
\mathbf{u}=\tilde{\mathbf{T}} \tilde{\mathbf{u}} .
$$


Finally, the element stiffness matrices $\mathbf{K}_{i}$ take the generalized displacements to the generalized forces (axial, shear, bending moment, and torsion moment). Block the element stiffness matrices so that the tensor $\mathbf{L}$ carries the displacement vector $\mathbf{u}$ to the strut forces

$$
\mathbf{F}=\mathbf{L}: \mathbf{u}
$$

with $\mathbf{F}$ the matrix of bar forces, $\mathbf{F}=\left[\begin{array}{llll}\mathbf{f}_{1} & \mathbf{f}_{2} & \cdots & \mathbf{f}_{n_{\text {struts }}}\end{array}\right]$. Combining Eqs. 32, 33, 34, 36, and 37 yields

$$
\mathbf{F}=\mathbf{L}: \tilde{\mathbf{T}} \mathbf{D}_{x f} \cdot \mathbf{E}: \mathbf{C}^{-1}: \boldsymbol{\sigma}
$$

which is a linear relation between the stress state and the bar forces. Finally, combining Eq. 38 with 31 completes the description of the failure surface.

For monotonic loading described by $\boldsymbol{\sigma}=a \hat{\boldsymbol{\sigma}}$, solving the failure function (Eq. 31)

$$
f(a \hat{\boldsymbol{\sigma}})=0
$$

for the load parameter $a$ determines the lattice strength in a given stress direction $\hat{\boldsymbol{\sigma}}$. Solving this equation repetitively for a uniform distribution of stress directions radiating from $\boldsymbol{\sigma}=\mathbf{0}$ gives a collection of points on the failure surface, approximately evenly distributed in stress space. These points can then be used to approximate the surface itself.

\subsection{Specific implementation}

A specific implementation of the general model outlined above requires a method of constructing a linear, undamped, homogeneous model for an arbitrary lattice unit cell as well as a set of limit state functions.

For this work, the implementation constructs the linear unit cell model from a standard frame element consisting of linear axial deformation, Euler-Bernoulli bending in two directions, and linear torsion. For linear analysis the mass and stiffness matrices fully define the element response. Appendix A describes the element. For the range of relative densities and vibrational frequencies considered here Euler-Bernoulli bending sufficiently describes the strut deformations for bend-dominated topologies. Higher relative densities or higher deformation frequencies may require a different bending theory, for example the Timoshenko theory, to account for shear distortion of the strut cross-sections and rotary inertia.

The implementation uses a single limit state function accounting for plastic bending and axial deformation 


\begin{tabular}{ccc}
\hline Property & Symbol & Value \\
\hline Young's modulus & $E$ & $113800 \mathrm{MPa}$ \\
Poisson's ratio & $\nu$ & 0.34 \\
Yield stress & $\sigma_{0}$ & $970 \mathrm{MPa}$ \\
Density & $\rho$ & $4.43 \mathrm{~g} / \mathrm{cm}^{3}$ \\
\hline
\end{tabular}

Table 1: Bulk material properties used in subsequent optimization problems.

coupled with buckling:

$$
l(\mathbf{f})= \begin{cases}\frac{|P|}{P_{\max }}+\frac{8}{9}\left(\frac{\left|M_{x}\right|}{M_{\max }}+\frac{\left|M_{y}\right|}{M_{\max }}\right) & \frac{|P|}{P_{\max }}<0.2 \\ \frac{1}{2} \frac{|P|}{P_{\max }}+\left(\frac{\left|M_{x}\right|}{M_{\max }}+\frac{\left|M_{y}\right|}{M_{\max }}\right) & \frac{|P|}{P_{\max }} \geq 0.2\end{cases}
$$

with $P$ the axial strut force, $M_{x}$ and $M_{y}$ the moments in the two bending directions,

$$
P_{\text {max }}= \begin{cases}A \min \left\{\sigma_{0}, \sigma_{e}\right\} & P<0 \\ A \sigma_{0} & P \geq 0\end{cases}
$$

with $A$ the strut cross-sectional area, $\sigma_{0}$ the bulk material yield stress, and

$$
\sigma_{e}=\frac{\pi^{2} E}{(K L / r)^{2}}
$$

the Euler buckling stress for the section - with $E$ the bulk material Young's modulus, $L$ the strut length, $r$ the section radius of gyration, and $K$ the column effective length factor - and

$$
M_{\text {max }}=Z \sigma_{0}
$$

with $Z$ the plastic section modulus. The AISC Steel Construction Manual [33] describes this limit state function in Chapter $\mathrm{H}$. The description here strips the safety factors from the interaction equation. However, according to the commentary, this interaction equation is somewhat conservative for circular cross sections. The equation accounts only for the interaction between axial plasticity, plastic bending, and buckling. Torsion and lateral-torsional buckling are not accounted for as they do not typically govern the response of symmetric cross sections.

Where possible the results below are non-dimensionalized so that the properties of the effective material can be presented independent of the bulk material constitutive response. Where this is not possible Table 1 describes the properties used for the bulk material. These properties describe additively manufactured Ti-6Al-4V . 


\section{Homogenized - - Bloch}

$\omega a / \sqrt{E / \rho}$

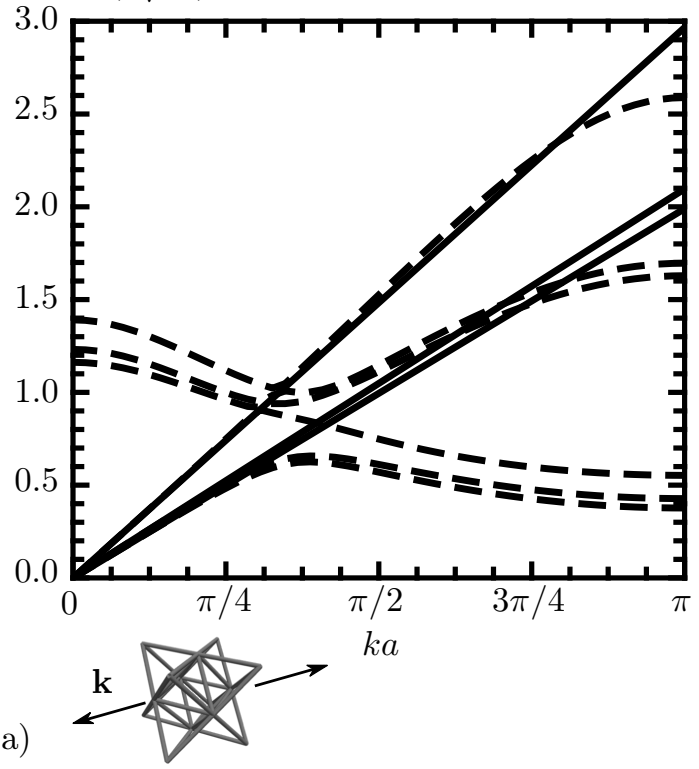

$\omega a / \sqrt{E / \rho}$

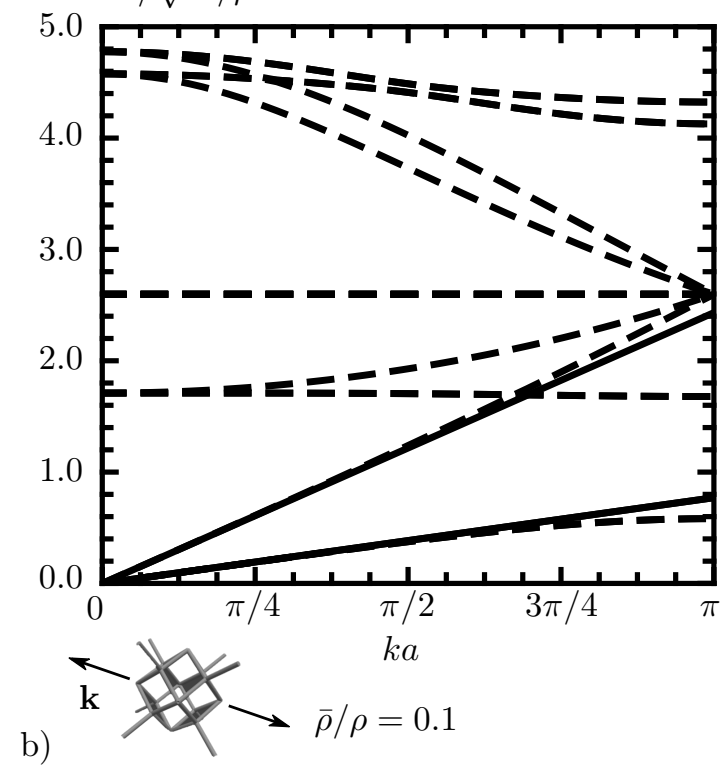

Figure 2: Dispersion relations for wavevectors in the indicated directions for the a) octet and b) rhombic-dodecahedron cells. The normalization of frequency by the thin rod bulk wavespeed $\sqrt{E / \rho}$ non-dimensionalizes the dispersion relation for the stretch-dominated octet lattice so that this non-dimensionalized dispersion relation applies for all values of relative density. The dynamics of the bend-dominated rhombic-dodecahedron depends on the combination of axial, bending, and torsional modes, so the dispersion relation cannot be properly non-dimensionalized. For the RD cell, this figure shows the relation for a relative density of $10 \%$.

\subsection{Verification}

\subsubsection{Convergence of finite size models to the periodic solution}

Figure 2 compares the dispersion relations predicted by the Bloch wave theory to the relations predicted by the long-wavelength homogenization. Figure 2a shows the dispersion relation for the stretch-dominated octet lattice while Fig. $2 \mathrm{~b}$ shows the relation for the bend-dominated rhombic dodecahedron lattice. The homogenization recovers the linearized dispersion relation around $k=0$ for the three lowest modes. For the lower modes the Bloch wave dispersion relation tends to be approximately linear. Therefore, the long wavelength homogenization accurately describes the response of the effective medium for low frequencies. The homogenization does not recover the higher order modes. A regular continuum theory cannot capture these modes, however a nonlocal or generalized continuum theory could approximate the higher modes. Researchers have developed such theories for anisotropic, locally inhomogeneous materials that share many characteristics with lattice-structured meta-materials. For example, see the work of Eringen and Edelen [34] and Eringen [35-37] on attenuation and dispersion in generalized elastic continua.

Figure 3 compares the stiffness and strength predicted by the current model to the stiffness and strength 

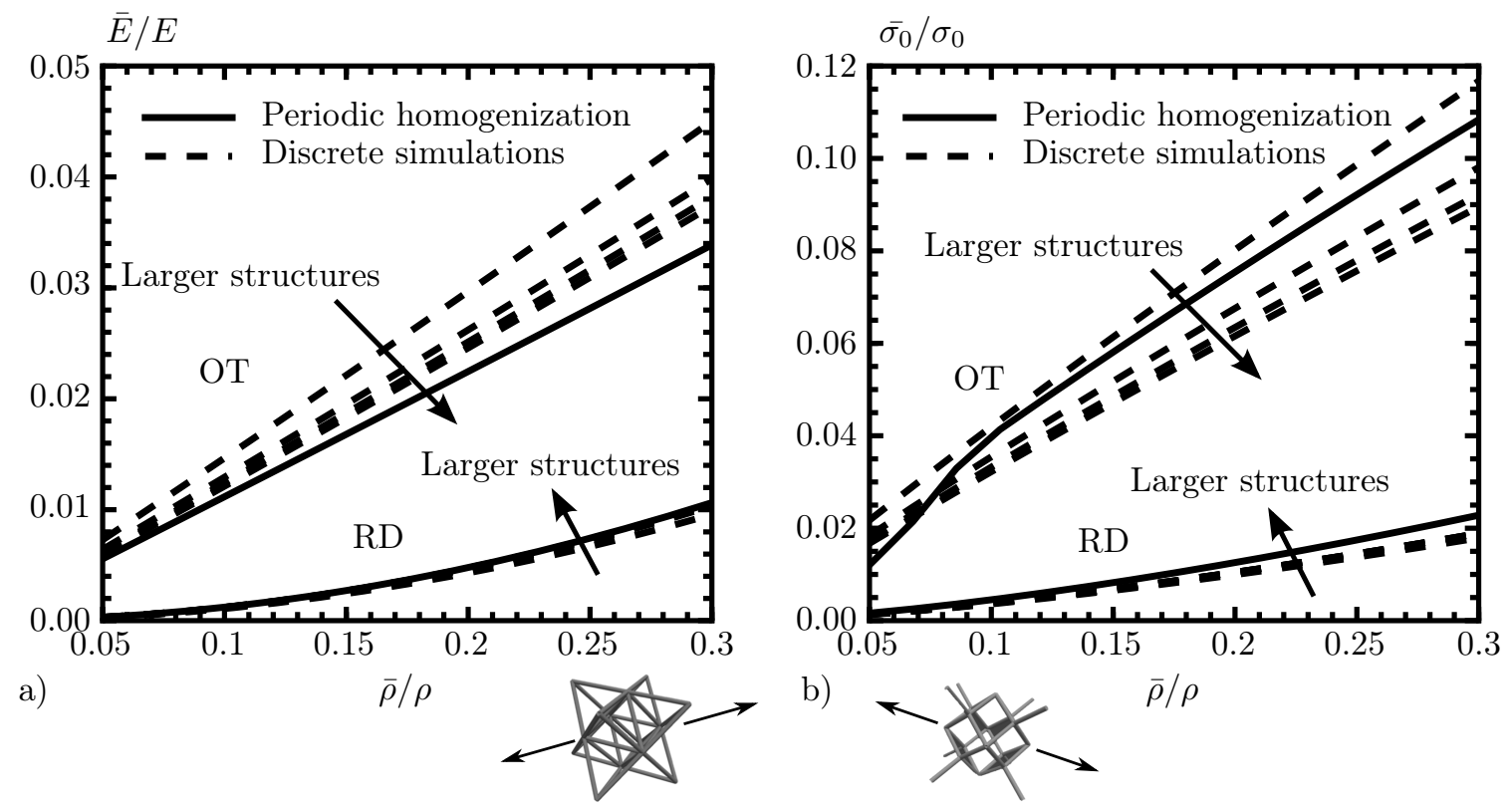

Figure 3: Plots demonstrating the convergence of the a) relative stiffness and b) relative strength of larger and larger discrete simulations $(2 \times 2 \times 2,4 \times 4 \times 4$, and $6 \times 6 \times 6$ unit cells) to the infinite periodic solutions. The figures show results for the stretch dominated octet truss cell (OT) and the bend dominated rhombic-dodecahedron (RD). The arrow indicates the direction of convergence: for the OT from above and for the RD from below. The sketch at the bottom of the figure indicates the loading direction.

calculated from finite element simulations of finite arrays of unit cells. The finite element simulations use the same frame element used to generate the linear unit cell model for the homogenized material description. The finite element simulations represent finite arrays of cells $(2 \times 2 \times 2,4 \times 4 \times 4$, and $6 \times 6 \times 6)$ for two different unit cells - again the stretch dominated octet truss and the bend dominated rhombic dodecahedron. The tiled arrays of cells are cubes because both of these unit cells have cubic symmetry. Cubic arrays make calculating the effective Young's modulus and compressive strength of the materials straightforward - the simulations apply a compressive traction, distributed as discrete nodal forces, to one face of the model while fixing the corresponding displacements on the opposite face. Of the remaining four faces two are constrained with symmetry boundary conditions and two are free. The models are one-eighth regions of a larger cubes of material, representing the remaining cells via the symmetry boundary conditions. With these boundary conditions a 1x1x1 simulated region represents a 2x2x2 block of material and so on. The effective compressive stress on the array is equal to the value of the applied traction and the effective compressive engineering strain is the average displacement of the traction face divided by the length of the cube. The effective Young's modulus of the cubic array is then the ratio of the stress to the strain at any load step. The model applies the compressive traction incrementally to compute the effective strength of the arrays. At each load increment the simulation evaluates the element forces in each of the 1D frame elements and then applies 


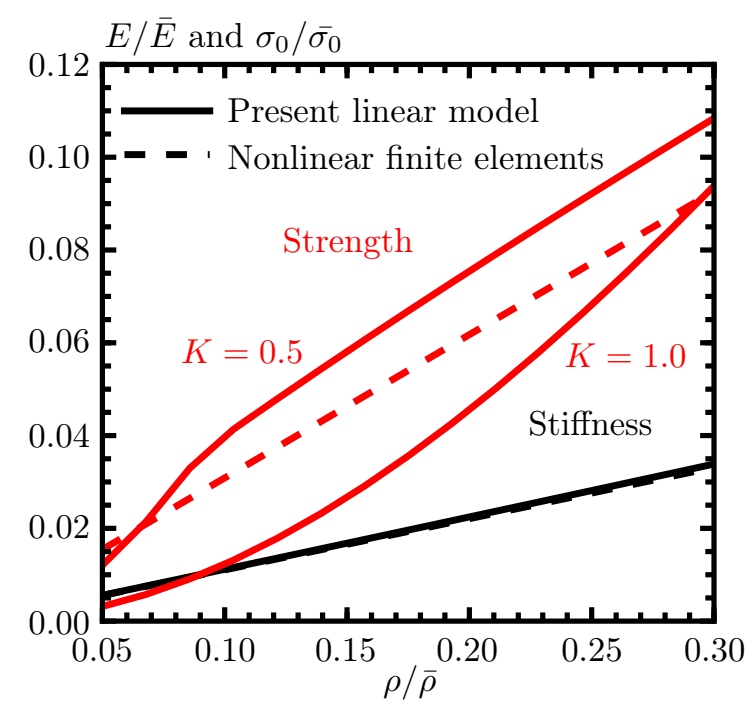

Figure 4: Figure comparing the scaling relations obtained via nonlinear finite element calculations (dashed) to those derived from the present model (solid). The figure shows both normalized stiffness (black) and strength (red) on the same axis. The two linear model strength lines represent two different end boundary conditions - fixed-fixed (upper) and pinned-pinned (lower).

the interaction equation (Eq. 40). This process is analogous to how the homogenized model computes the effective strength from the forces in the periodic unit cell. The figure shows the results of multiple simulations of each topology at different densities in order to capture the scaling relation between stiffness/strength and relative density.

The figure shows convergence in the elastic stiffness. As the size of the discrete array finite element simulations increases the scaling relation converges to that predicted by the homogenized model. However, the strengths calculated from the finite element simulations do not converge to the predicted values. Edge effects, which are not included in the homogenized model representing an infinite, periodic array of unit cells, cause this discrepancy. Further discussion appears below.

\subsubsection{Comparison to nonlinear calculations}

Figure 4 shows the scaling relations for stiffness and peak compressive strength versus relative density for a large 16x16x16 cubic array of OT cells computed via a nonlinear finite element calculation. The figure compares these relations to the scaling relations calculated with the current model. The FE calculation uses nonlinear bar elements considering both material nonlinearity, in the form of a bilinear plastic material model with properties given by Table 1 and supplemented with plastic hardening modulus $H=1000 \mathrm{MPa}$, and geometric nonlinearity caused by the large displacements of the node positions. Note the comparison between the models is not exact - the linear periodic model allows for bending deformation in the members while the nonlinear model does not. For stretch dominated topologies like the OT there is no difference 
between the formulations for the effective elastic stiffness. However, the calculated limit strengths depend on the underlying structural formulation.

The figure shows two strength scaling lines for the linear model - one with $K=1.0$ (pinned-pinned strut boundary conditions) and one with $K=0.5$, used throughout this work (fixed-fixed boundary conditions). For low relative densities, where the approximations of 1D element modeling are most accurate, the two strength curves bracket the nonlinear bar solution. The curve corresponding to the actual nonlinear simulation boundary conditions (pinned-pinned) under predicts the strength because the interaction equations are designed to be conservative and because the nonlinear model does not account for bending deformation induced by large deformations changing the initially stretch-dominated topology of the unit cells.

\section{Microstructural optimization}

\subsection{Parameterization of the design space}

The previous section describes a model for the homogenized mechanical response of a periodic lattice structure. The input to the homogenized model is a linear representation of the microscale deformation of a single unit cell. Applying numerical optimization methods to find optimal microstructures requires a parameterized description of the design space - a way to describe all possible lattice unit cells. Ideally, such a parameterization would be a compact vector of real numbers - avoiding the difficulty inherent in optimizing integer parameters and minimizing the number of design variables. This section describes such a parameterization with one limitation: the description considers all lattices with degree $d$ or less (see Section 2).

Four kinds of parameters are required to describe a unit cell:

1. a description of the simple lattice $\left(J_{0}\right)$ joint positions,

2. a description of the position of the remaining joint sets, naturally described as the set of joint basis vectors,

3. a description of the struts connecting the joints,

4. and (optionally) a rotation describing the orientation of the unit cell relative to a global coordinate system.

A Bravais lattice describes the position of the simple lattice joints. In 2D, two lengths and one angle describe a Bravais lattice. In 3D, three lengths and three angles are required. The lattice itself fixes the position of the primitive cell joints in joint set $J_{0}$. The joint basis describes the positions of the remaining joints in $J_{1}, J_{2}$, ... Since the points described by these vectors must fall inside the primitive unit cell they can be described by fractional lattice coordinates. In $2 \mathrm{D}$, two real numbers in the range $[0,1]$ parameterize each point in the 


\begin{tabular}{cccccc}
\hline Dimension & Simple lattice & Basis vectors & Strut areas & [Orientation] & Total \\
\hline 2D & 3 & $2 d$ & $(8+d(7+d)) / 2$ & {$[1]$} & $(14+d(11+d)) / 2+[1]$ \\
3D & 6 & $3 d$ & $((2+d)(13+d)) / 2$ & {$[3]$} & $((2+d)(19+d)) / 2+[3]$ \\
\hline
\end{tabular}

Table 2: Summary of the number of parameters required to describe the lattice design space in 2D and 3D, given the lattice degree $d$. The numbers in brackets account for the parameters needed to describe the orientation of the unit cell.

joint basis. In 3D, three real numbers are required for each point. To avoid integer parameter optimization the method poses the optimization problem in terms of the lattice degree $d$. Selecting the degree fixes the number of joint basis vectors to $d$.

Given the position of the joints in the primitive cell, the parameterization considers all possible struts connecting the set of joints. The parameterization describes the struts in terms of their cross-sectional areas. Given the geometrical shape of the cross-section the optimization method can convert these cross-sectional areas to the additional parameters required for the microscale model, primarily moments of inertia. This work assumes the struts have solid circular cross-sections.

However, not all the strut cross-sectional areas are free parameters. Certain struts - those on the edges of the unit cell in 2D and the edges and faces of the unit cell in 3D - are translationally equivalent. These struts must have the same areas. This requires a translation step to take a reduced set of cross-sectional areas to the complete set of areas describing each strut in the unit cell. Similarly, struts on the edges or faces of the unit cell have reduced cross-sections accounting for material shared with the neighboring cell(s). The conversion from strut area to linear model must also account for this variation in the strut section shape.

For general structural optimization problems the spatial orientation of the lattice needs to be parameterized. For example, if the optimization problem was to find the optimal lattice structure at each point in some spatial domain then the rotation of the lattice at each spatial point, relative to the global coordinates, affects the response of the structure. In 2D one parameter describes the lattice orientation; 3D optimization requires three parameters.

Table 2 summarizes the number of parameters, relative to the lattice degree $d$, for two- and threedimensions. The number of parameters increases quadratically with $d$, making it more feasible to look for simple lattices or complex lattices with low degree. Let $\mathbb{L}_{d}$ be the complete set of all possible lattices with degree $\leq d$. Call a point in the space $\mathcal{L} \in \mathbb{L}_{d}$, which represents a particular lattice topology. The optimization problems posed here seek the lattice $\mathcal{L} \in \mathbb{L}_{d}$ that minimizes a given objective function $R(\mathcal{L})$, subject to constraints $c_{i}(\mathcal{L})$.

This parameterization does not prevent infeasible structures with intersecting struts not meeting at a model joint. These struts would physically cross but do not interact in the resulting unit cell model. A filter avoids this unphysical possibility by only allowing one of the intersecting struts to pass into the discretized 
cell model. It sets the cross-sectional area of the other strut to zero. In 3D there six classes of non-physical, intersecting struts on the primitive cell faces and one class of possible intersections in the interior of the cell.

This parameterization requires an optimization method that accepts bounds on the parameters. The basis vectors, expressed as fractional lattice coordinates, must have components in the range $[0,1]$ and the strut cross-sectional areas must be greater than or equal to zero. Bounds are not required on the remaining parameters, however they can be imposed to produce more practical, buildable structures. The subsequent optimization problems tie the lattice parameters to a feasible manufacturing length scale. The first lattice parameter, $a_{1}$ is fixed to $4 \mathrm{~mm}$ - a reasonable unit cell scale for the selective laser melting process. The optimizer can alter the remaining two lattice parameters such that $a_{1} / 10<a_{2}<10 a_{1}$ and $a_{1} / 10<a_{3}<10 a_{1}$.

\subsection{Optimization method}

The parameterized model, objective functions, constraints, and bounds on the parameters are fed to the sequential least square programming (SLSQP) [38] optimizer embedded in the Scipy python package [39]. The optimizer computes the sensitivities of the objective function and the constraints via finite differencing feasible because the design space, as described with the above parameterization, is small. A custom Python script reconstructs the optimal parameter sets into lattice unit cells for display.

\subsection{Optimal lattices}

\subsubsection{Isotropic elastic stiffness}

An ideal elastic lattice material would be maximally stiff and maximally isotropic. The norm of the effective stiffness tensor, relative to the bulk material Young's modulus and the density of the lattice, provides a general measure of elastic stiffness:

$$
R_{\text {stiff }}=\frac{\|\overline{\mathbf{C}}\|}{\rho E} .
$$

Ranganathan et al. [40] propose a general measure of elastic anisotropy, given as

$$
A_{U}=5 \frac{G_{V}}{G_{R}}+\frac{K_{V}}{K_{R}}-6
$$

with $G_{V}$ and $K_{V}$ the Voigt bound shear modulus and bulk modulus and $G_{R}$ and $K_{R}$ the Reuss bound shear modulus and bulk modulus. With this measure $A_{U}=0$ indicates elastic isotropy, with higher values of $A_{U}$ being more anisotropic. The objective and constraint functions are frame invariant, so the orientation of the lattice relative to the coordinate system will not influence the optimization results. 


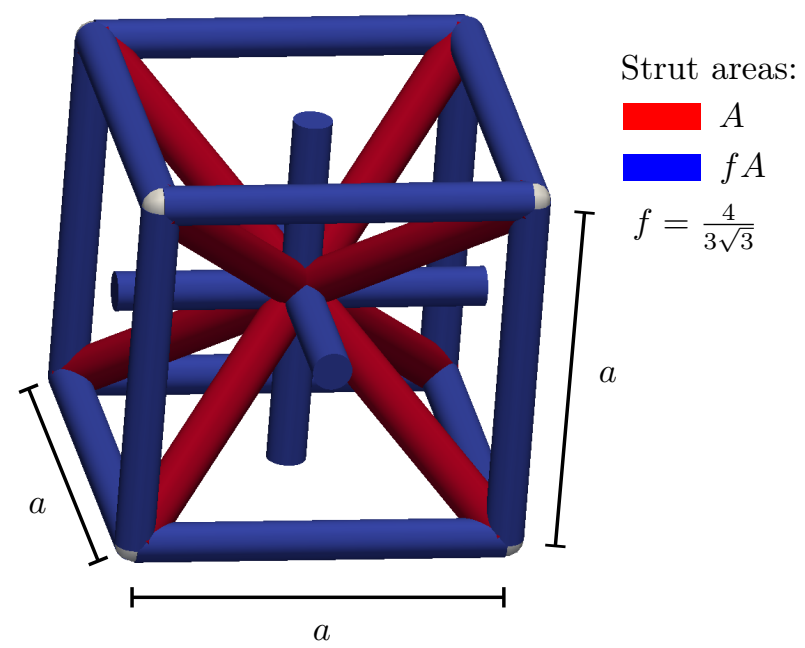

Figure 5: The isotruss unit cell topology. This shows the simple cubic cell, rather than the primitive BCC cell described by the optimized parameters. The cross sectional areas of the two classes of struts are drawn to scale and also highlighted in red and blue.

The optimization problem

$$
\begin{array}{r}
\operatorname{maximize} R_{\text {stiff }}(\mathcal{L}) \\
\text { subject to } A_{U}(\mathcal{L})=0
\end{array}
$$

produces an optimally stiff, isotropic lattice. For an isotropic material $\|\mathbf{C}\|=3 K$, with $K$ the bulk modulus, so maximizing $\|\mathbf{C}\| /(\bar{\rho} E)$ is equivalent to maximizing the relative bulk modulus. The numerical optimization problem imposes the equality constraint as an inequality with a small tolerance.

For $d \in[0,3]$ the optimization problem converges to the same BCC, simple, stretch dominated cell, shown in Fig. 5 as a simple cubic cell. The structure has two different classes of struts with different lengths and cross-sectional areas. Because the lattice is elastically isotropic and stretch-dominated the remainder of the work refers to it as the isotruss. The ratio between the strut areas is $f=4 /(3 \sqrt{3})$ and the formula relating the strut areas and cubic cell size to the relative density is $\bar{\rho}=\frac{20 A}{\sqrt{3} a^{2}}$. Analyzed as a truss lattice with the 
method presented in [26] its effective stiffness matrix is

$$
\mathbf{C}=\frac{E \bar{\rho}}{15}\left[\begin{array}{cccccc}
3 & 1 & 1 & 0 & 0 & 0 \\
1 & 3 & 1 & 0 & 0 & 0 \\
1 & 1 & 3 & 0 & 0 & 0 \\
0 & 0 & 0 & 1 & 0 & 0 \\
0 & 0 & 0 & 0 & 1 & 0 \\
0 & 0 & 0 & 0 & 0 & 1
\end{array}\right]
$$

This lattice has $\bar{E} / E=\bar{\rho} / 6, \bar{\nu}=1 / 4$, and $\bar{\mu} / E=\bar{\rho} / 15$ with $\bar{\mu}=\bar{E} /[2(1+\bar{\nu})]$ and is therefore elastically isotropic. The bulk modulus scales as $\bar{K} / K=\bar{\rho}(1-2 \nu) / 3$.

\subsubsection{Maximally-isotropic strength}

A useful lattice material might have a failure surface that resembles the yield surface of conventional bulk materials. A von Mises yield surface approximates the elastic limit of many standard isotropic materials. This optimization example tries to find a lattice material with a failure surface as close to von Mises as possible.

Finding such a structure requires an objective function describing similarity between an arbitrary failure surface and the surface produced by the von Mises criterion. In principal stress space the von Mises surface is a right circular cylinder centered on the hydrostatic axis. The maximum cylindrical deviation from the volumetric axis is then a reasonable measure to compare arbitrary surfaces to the von Mises surface. Let $r\left(\sigma_{M}\right)$ be the function describing the distance between the volumetric axis and the yield surface in the deviatoric plane ( $\Pi$ plane) centered at $\frac{1}{3} \operatorname{tr}(\boldsymbol{\sigma})=\sigma_{M}$. For each $\Pi$ plane let $r_{i}=\max r$ - the radius of the inscribed circle - and $r_{c}=\min r$ - the radius of the circumscribed circle. Figure 6 illustrates this idea for a non-Mises surface.

For a given value of mean stress, the ratio

$$
M\left(\sigma_{M}\right)=1-\frac{r_{c}\left(\sigma_{M}\right)}{r_{i}\left(\sigma_{M}\right)}
$$

describes how circular the surface is on the $\Pi$ plane passing through mean stress $\sigma_{M}$. Lower values of $M$ indicate more circular failure surface cross-sections. The maximum value of $M$ across the entire hydrostatic axis describes the similarity of the failure surface to the von Mises surface

$$
M=\max _{\sigma_{M}} M\left(\sigma_{M}\right) .
$$




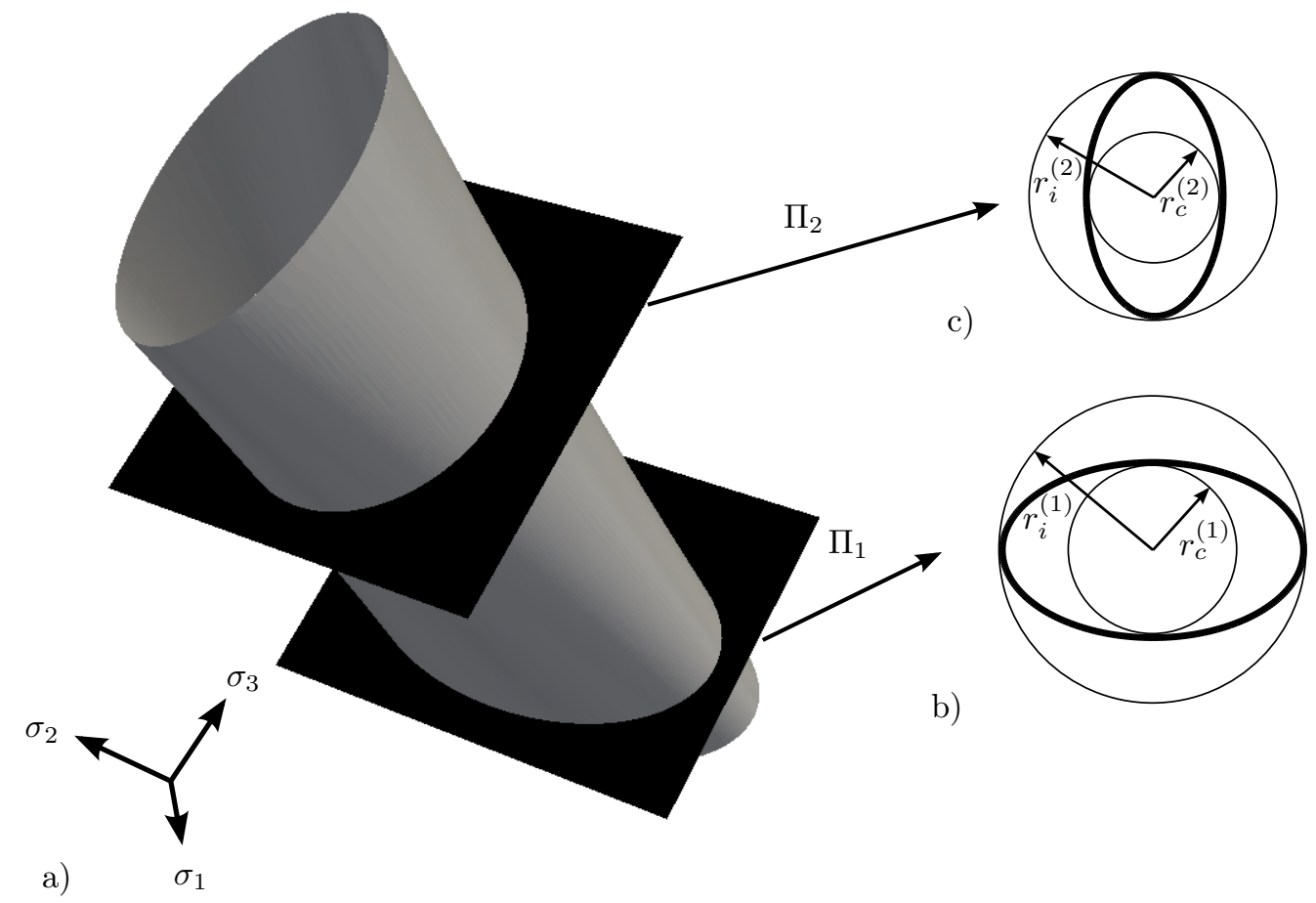

Figure 6: This figure illustrates the method used to compare failure surfaces to the von Mises yield surface. a) An example failure surface in principal stress space. b) A slice on a deviatoric $\Pi$ plane, positioned at a particular value of mean stress. c) A different slice on a different $\Pi$ plane. b) and c) show the radii of the inscribed and circumscribed circles, used to make the quantitative comparison.

The von Mises surface has $M=0$. Note this criteria only addresses local similarity to the von Mises surface on each $\Pi$ plane. For example, a surface that has a circular cross section with a different radius at each level of $\sigma_{M}$ would also have $M=0$. However, such a surface still locally resembles the von Mises surfaces for all values of hydrostatic stress.

Failure surfaces for lattice materials have caps. That is, these materials will yield even in pure hydrostatic stress. A lattice material will therefore never exactly reproduce the uncapped von Mises surface, which extends to infinity in both directions along the hydrostatic axis. Equation 49 is undefined for surfaces with caps because at some point along the hydrostatic axis the radii $r_{i}$ and $r_{c}$ become undefined - the surface has no intersection with the $\Pi$ plane. Therefore, this optimization problem only looks for lattice materials which resemble the von Mises surface around $\sigma_{M}=0$. In particular, we restate Eq. 49 as

$$
M_{C}=\max _{\sigma_{M} \in\left[-\sigma_{Y} / 75, \sigma_{Y} / 75\right]} M\left(\sigma_{M}\right)
$$

to limit the search for the maximum deviation from a cylinder to the $\Pi$ planes with mean stresses in the range 


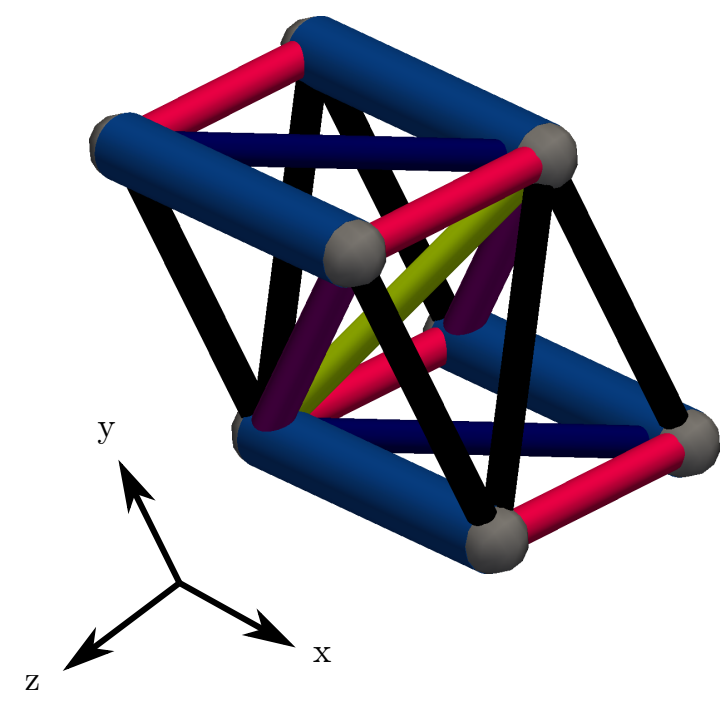

Figure 7: The lattice structure produced by the optimization problem described in Eq. 51. Different colors indicate struts with different cross-sectional areas, which are also drawn to scale.

[- $\left.\sigma_{Y} / 75, \sigma_{Y} / 75\right]$. Surfaces with low values of $M_{C}$ will resemble a von Mises surface under low hydrostatic stress.

The optimization problem

$$
\begin{array}{r}
\operatorname{mimimize} M_{C}(\mathcal{L}) \\
\text { subject to } \bar{\rho}(\mathcal{L})=0.1 \\
\bar{\sigma}_{0}^{(x x)}>\sigma_{0} / 75,
\end{array}
$$

with $\bar{\sigma}_{0}^{(x x)}$ the limit stress for uniaxial tension in the $x$-direction, yields a $10 \%$ dense lattice topology with a failure surface that most resembles the von Mises yield surface while avoiding structures with negligible strength. The final constraint prevents the optimizer from finding structures with no strength in any direction - an isotropic but spurious solution. However, because it imposes a strength requirement in a particular direction in stress space, the resulting optimization problem is not frame invariant. Posing a minimum strength requirement in a different direction could produce a different structure. Figure 7 shows the simple lattice structure produced by this optimization problem.

Figure 8 shows the failure surface for the optimal lattice, with two deviatoric slices showing the deviation of the surface from a circle. Several factors limit the ability of a lattice material to reproduce the smooth von Mises surface. These limitations are discussed below. 


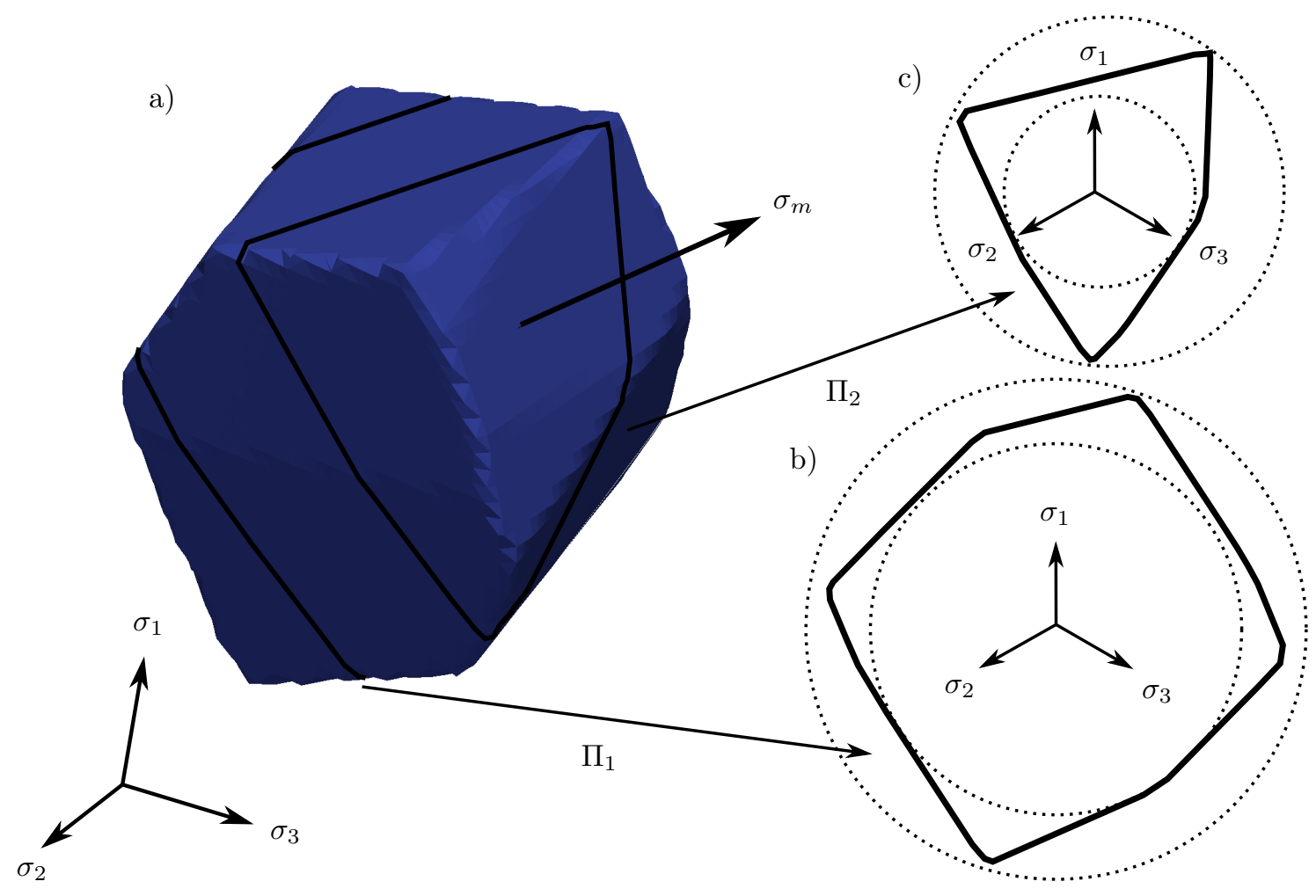

Figure 8: a) The failure surface of the optimal lattice material. b) A deviatoric slice of the surface at $\sigma_{M}=0-$ the principal $\Pi$ plane. c) A similar slice at an arbitrary, larger, tensile value of $\sigma_{M}$. 




Figure 9: A sketch of a finite-size $(6 \times 6 \times 6)$ octet truss lattice material highlighting the failed struts for uniaxial compression loading on one of the array faces. The red struts failed - that is, $f(\boldsymbol{\sigma})=0$ at this stress state. Notice the failed struts are all adjacent to the outside faces of the array. This pattern holds true for an array of any size - the critical members are always on the boundary of the material. The blue box outlines the edges of the cubic array of cells - the blue lines are not struts in the simulation.

\section{Discussion}

\subsection{Effective properties of lattice structures}

Figures 2 and 3 demonstrate the convergence of the method for analyzing the linear elastic response of lattice materials proposed in this work. Figure 2 shows the homogenized analysis reproduces the dynamics of the exact, Bloch wave solution in the limit of long wavelengths. This implies the homogenization will accurately represent long wavelength, low frequency dynamics in lattice materials. Figure 3a shows the elastic response of finite lattice materials rapidly converges to the infinite, periodic response as the physical size of the structure, in terms of number of unit cells, increases. This implies that, given an accurate characterization of the bulk material and the microscale deformation, the homogenized analysis proposed here will be accurate for printed structures with more than about eight unit cells in all directions. Current technologies produce lattices with unit cell sizes ranging from a few millimeters to a few microns [1-3], so most applications of lattice materials will include at least several hundred unit cells.

The strengths of the finite-size structures in Fig. 3b do not converge exactly to the infinite, periodic solution generated by the homogenized model. Figure 9 illustrates the reason for this failure. As the figure shows, the critical struts - the first to fail - always occur on the boundaries of the finite-size lattice materials. These struts are strongly affected by boundary effects that are not present in the infinite, periodic 
homogenization. These boundary effects also affect the elastic properties of the material and explain why the stiffness of a small array of unit cells does not match the infinite solution. However, for the elastic properties, as the size of the finite-size lattice increases the boundary effects are averaged over a larger and larger volume of material. Therefore, the elastic properties of the finite structures converge to the infinite solution. In contrast, failure, as defined in this work, is a weakest-link process. Given this definition, the response of finite size structures will never converge to the infinite solution, no matter how large the periodic array of unit cells becomes. The array will always have boundaries, which are never accounted for in the periodic homogenization.

Two factors offset this shortcoming of the homogenized model. First, while the homogenized model does not exactly capture the response of finite-sized lattice materials, the infinite solution does approximate the failure stress of larger finite-size materials. A pragmatic approach to using the model developed in this work to predict the strength of actual structures might be to apply a safety factor to the model predictions. Secondly, for large finite-size structures, the definition of failure proposed in this work becomes less useful. This work defines failure as a single strut failing. For a large structure a single strut failure on the edge of the material volume would essentially be unnoticeable in the macroscale, effective response of the material as a whole. For very large structures a better definition of failure might be the yielding or buckling of some critical region of material, perhaps of a few unit cells. With this definition of failure the surface predicted by the periodic homogenization would be much more accurate.

The previous sections discuss a further two, related shortcomings of the homogenized model. The homogenized response does not predict the higher order modes or the attenuation and dispersion found in the dynamics of the exact Bloch wave solutions. Similarly, the model does not capture the effect of local buckling modes that coordinate over a finite number of unit cells. Section 2.3 outlines how to capture these local modes using nonlinear analysis of unit cells. This work excludes both of these effects in order to develop a homogenized model suitable for optimization. Such a model must be computationally efficient in order to quickly evaluate trial structures inside the optimization loop.

\subsection{Optimal structures}

Figure 10 plots the Young's modulus for the optimally stiff lattice against the Hashin-Shtrikman upper bound for a composite of elastic material with Young's modulus $E$ and Poisson's ratio $\nu=0.25$ and void [41]. For comparison the figure also plots the scaling relation for the octet truss for loading along one of its simple cubic axes (see the sketches in Figs. 2 and 3, scaling relation $\bar{E} / E=\bar{\rho} / 9$ ) and along the direction of maximum stiffness $(\bar{E} / E=\bar{\rho} / 5)$ [27]. The structure found in this work outperforms the octet in its least-stiff direction and only narrowly underperforms the octet along its stiffest direction. More importantly, 


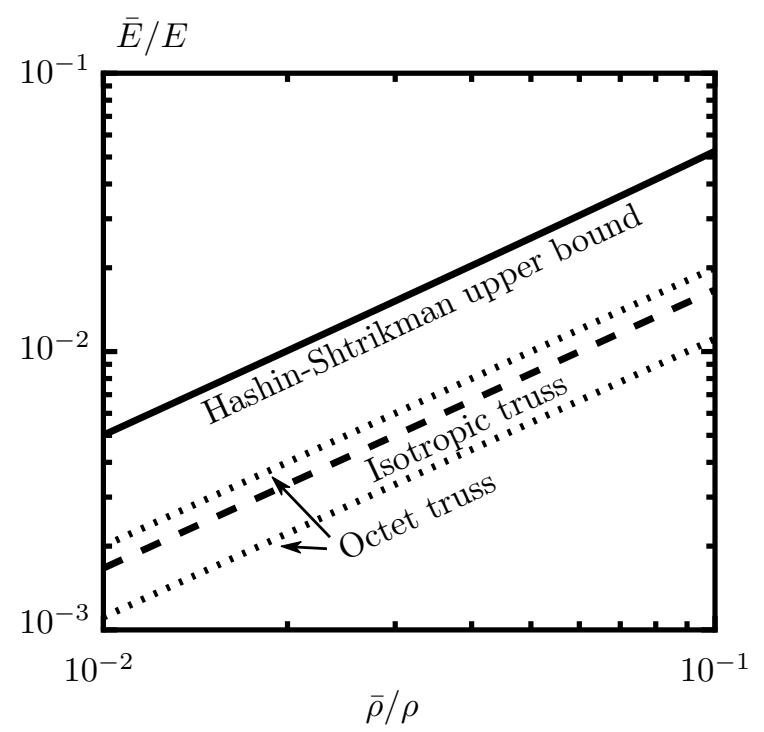

Figure 10: Relative stiffness-to-relative density scaling relation for: 1) the Hashin-Shtrikman upper bound, 2) the octet truss, in the directions of maximum and minimum stiffness, and 3) the isotruss lattice found in this work.

the structure found here is elastically isotropic while the octet has cubic anisotropy with anisotropy ratio $A=2$. Both the isotropic truss and the octet approach $50 \%$ of the theoretical upper bound on the Young's modulus.

The isotruss structure found here is arguably more optimal than the octet truss, which currently appears to be the standard stretch dominated unit cell used in the literature (e.g. $[1,6,42])$. The least-stiff direction in the octet is the lattice direction for a simple cubic unit cell. This means many printed structures align the loading with the least stiff direction of the microstructure. The optimal structure found here outperforms the octet by $50 \%$ in this direction. Furthermore, the isotruss is elastically isotropic. Design and analysis of structures using this cell can decouple the response of the material in different directions. This makes analysis of large structures using the isotruss cell somewhat less difficult than for the octet - the analysis method requires no special treatment for anisotropy. Finally, unlike the octet, the elastic stiffness of the isotruss is independent of the orientation of the unit cell. This means no special analysis is required for structures where the lattice microstructure is printed conformally with some curved macroscale feature. For the octet in this situation the analysis would have to account for the variation of the material axes across the structure.

The difference in the strut cross-sectional areas for the isotruss unit cell should not pose a challenge to constructing a lattice material using any AM process that allows for such variations - for example selective laser melting and micro-stererolithography. The ratio between the struts areas is $f \approx 0.77$, so the length scales of the two strut classes are not widely disparate. However, processes with no control over the cross 
sections of individual members - such as direct ink write processes - could not assemble the isotruss structure. Potentially for such processes the stiffness of the material could be varied instead of the cross-section. The build processes could use two different materials or vary the processing parameters to generate the different elastic stiffnesses.

The maximally-isotropic strength criterion produces a less optimal structure than the maximally-isotropic stiffness criterion. The effective failure surface for the optimal material (Fig. 8) is moderately isotropic only near the principal $\Pi$ plane at $\sigma_{M}=0$. Two factor limit the ability of a lattice structure to mimic a von Mises surface. The first factor is tension-compression asymmetry. In compression, slender struts can yield or buckle; in tension yielding is the the only failure criterion considered here. The optimizer cannot fully correct for this microscale asymmetry with the topology of the unit cell. The second factor is the discrete nature of failure in lattice materials. The criteria adopted for this work defines failure of the material as the stress state at which the first strut fails. This is an inherently discrete criteria - for any structure there is likely to be a range of stresses that produce failure in a given strut. The flat (or straight, in-cross section) facets of the optimal failure surface reflect this discrete character. These flat facets describe regions in stress space governed by the response of a single class of periodic struts. To some extent lattice materials cannot avoid this faceted description of the failure surface - by definition any lattice material is a discrete collection of struts, each of which could generate a facet on the failure surface. More complex lattices, with more classes of geometrically-distinct struts, could potentially smooth the surface to a greater extent by better approximating a circle with a larger number of straight segments.

\subsection{Elastic boundary layers}

Elastic boundary layers develop near the free surfaces of lattice materials to account for the transition between the uniform stress state in the interior of the material and the traction-free boundary conditions on the edge. If these boundary layers grow with the size of the material - in terms of number of unit cells they can adversely affect the effective elastic properties of the material and produce experimental size effects. Phani and Fleck [43] developed an elegant way to examine the size of such boundary layers using Bloch wave theory. This work analyzes these boundary layers in a more direct manner - by examining the behavior of large finite-size models near a free surface.

As described by Phani and Fleck [43], a Bloch attenuation wave in the finite-size lattice structures defines the elastic boundary layer in the octet and isotruss. Exponential decay from the boundary stress state to the uniform center stress characterizes the boundary layer. Figure 11 describes the size of this boundary layer and its effect on the effective elastic properties of finite-size structures with the following procedure:

1. Setup a finite-size simulation of a cubic array of cubic octet and isotruss unit cells. These simulations 

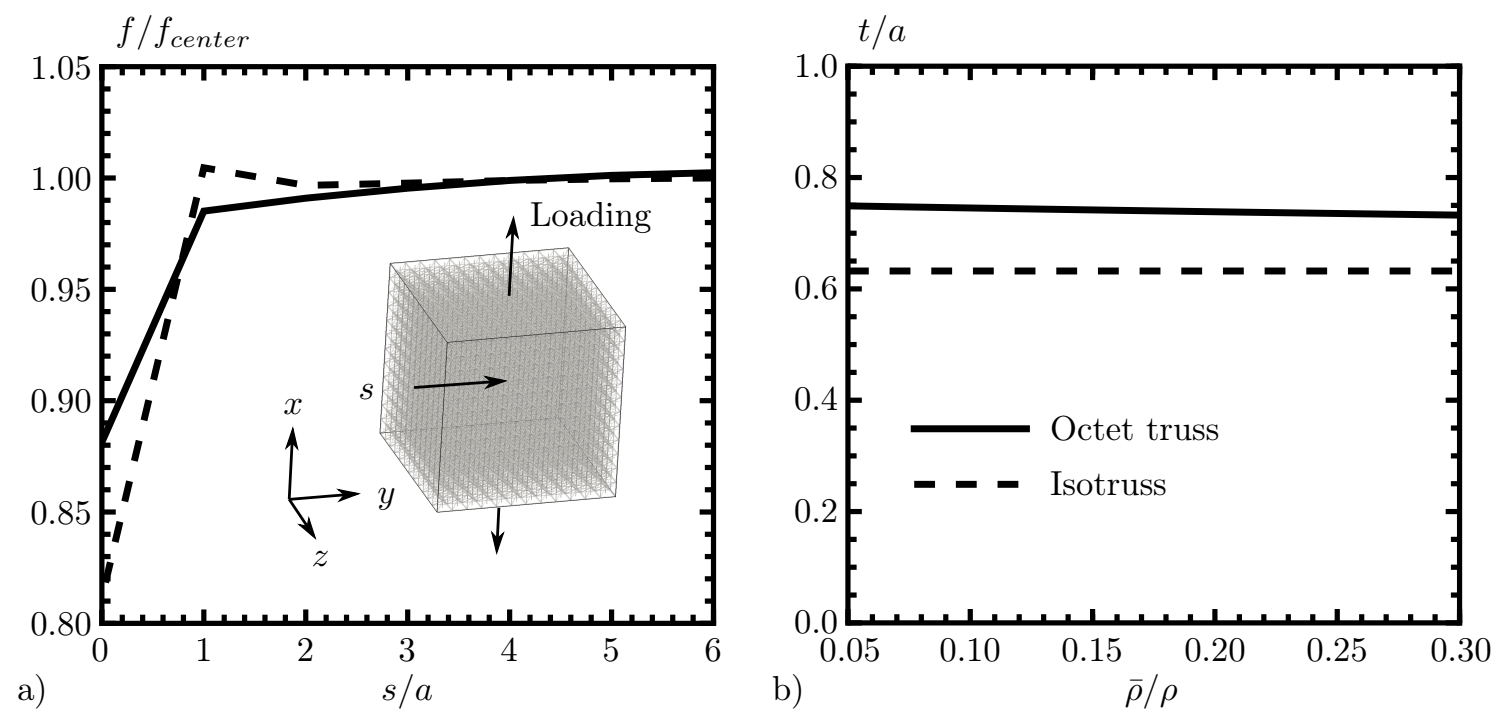

Figure 11: Plots illustrating the development of an elastic boundary layer on the free surface of a finite size lattice. Solid lines are results for octet truss structures and dashed lines are results for isotruss structures. a) Typical force versus distance from free surface curves for both unit cell types. Force values are normalized by the force at the center of the model. This instance is for $10 \%$ relative density. b) Normalized thickness of the elastic boundary layer versus relative density.

are of the same type as in Section 2.5.1 used to characterize the convergence of finite size simulations to the long wavelength homogenization. The simulations used for these elastic boundary layer calculations are $12 \times 12 \times 12$ arrays of unit cells.

2. Apply a uniform displacement to the top face of the model in the positive $x$-direction.

3. Plot the $x$-direction forces at the model joints on a line starting from the edge of the model and extending in the $y$-direction to the model center (see diagram in Fig. 11a). This produces a curve of the kind shown in Fig. 11a.

4. Characterize the size of the boundary layer as the distance from the free surface, in terms of the unit cell size, at which the nodal forces achieve $1-1 / e \approx 0.632$ of force difference between edge node and the center node forces.

5. Repeat this procedure for several values of relative density.

This series of calculations was carried out for both the isotruss and octet cells. Figure 11b summarizes the results: for both topologies the elastic boundary layer stays confined to the first unit cell inside from the free boundary and, for both topologies, the size of the boundary layer does not change with the relative density of the lattice. This places both topologies in the "shallow" boundary layer category developed by Phani and Fleck, indicating the effect of boundary layers will be negligible for large structures for both types of cells. 


\section{Conclusions}

The primary contributions of this work are:

1. a homogenized model for the long-wavelength elastic and failure properties of arbitrary lattice materials;

2. a compact, intrinsically-periodic parameterization of the design space of lattice structures;

3. and a method for using the resulting parameterized model to optimize mesoarchitectures.

The homogenized model represents the long wavelength limit of the discrete, microscale lattice dynamics, embedded in a conventional continuum formulation. As such, the homogenized model only accurately captures response of the lattice for low frequency deformation and the model neglects local buckling modes.

The optimized structures presented here are examples of how optimization methods can use the parameterized model to find useful mesoarchitectures. The optimally stiff isotruss lattice in particular improves upon the octet truss topology as it has a superior stiffness-to-density ratio while being elastically isotropic. This structure, and other isotropic structures, greatly facilitate the analysis of conforming lattices where the lattice topology rotates to conform to some macroscale design feature. The same method could also be used to optimize topologies for particular applications with given stiffness and/or strength requirements.

The compact parameterization of the lattice design space developed here means the optimization problems in this work involve fewer than 100 design variables. Therefore, this work does not focus on the optimization methodology, using a simple gradient-based scheme with a finite-differenced gradient. Future work should address the optimization methodology in order to assure good convergence to optimal solutions for nonconvex objective functions. This future work could either address the conditioning of gradient-based methods by analytically computing and examining the gradients of the objective functions and constraints or by applying gradient free optimization methods. See [44] and [45] for two examples of applying gradient-free methods to non-periodic truss/frame optimization problems with good results.

Given the parameterized model a logical next step is to solve a structural optimization problem to find the optimal lattice structure at each point in a spatial domain for some global objective function. Nakshatrala and Tortorelli [46] and Le et al. [18] demonstrated success optimizing microstructures to direct dynamic energy though a material. Their approach could now be applied to the parameterized lattice model to generate printable structures. Formulating an optimization problem to generate lattice materials that are constructible may require additional constraints to eliminate structures with valid unit cells - achieved by the filter described in this work - but invalid placement. For example, their must be some smoothness constraint on the parameters describing the lattice primitive unit cell or adjacent cells in the optimized structure could be incompatible - leaving a gap or an overlap between cells. Once these constructibility requirements are 
fulfilled the resulting optimization methods have the potential to control both the lattice anisotropy and inhomogeneity over a spatial domain to find optimal, lightweight, printable lattice structures.

\section{Acknowledgments}

This work was performed under the auspices of the U.S. Department of Energy by Lawrence Livermore National Laboratory under Contract DE-AC52-07NA27344 (LLNL-JRNL-683819). The author thanks Holly Carlton for providing the image of a lattice material, used as Fig. 1, and Nathan Barton for a productive discussion on how to measure similarity to the von Mises yield surface.

\section{References}

[1] X. Zheng, H. Lee, T. H. Weisgraber, M. Shusteff, J. DeOtte, E. B. Duoss, J. D. Kuntz, M. M. Biener, Q. Ge, J. A. Jackson, S. O. Kucheyev, N. X. Fang, C. M. Spadaccini, Ultralight, ultrastiff mechanical metamaterials., Science 344 (2014) 1373-1377. doi:10.1126/science.1252291.

URL http://www.ncbi.nlm.nih.gov/pubmed/24948733

[2] T. A. Schaedler, A. J. Jacobsen, A. Torrents, A. E. Sorensen, J. Lian, J. R. Greer, L. Valdevit, W. B. Carter, Ultralight Metallic Microlattices, Science 334 (2011) 962-965.

URL http://www.sciencemag.org/content/334/6058/962.full

[3] C. Yan, L. Hao, A. Hussein, D. Raymont, Evaluations of cellular lattice structures manufactured using selective laser melting, Int. J. Mach. Tools Manuf. 62 (2012) 32-38.

URL http://dx.doi.org/10.1016/j.ijmachtools.2012.06.002

[4] H. C. Guo, X. M. Qiu, D. N. Fang, Optimal design of two lattice materials and their effective properties, Int. J. Nonlinear Scienes Numer. Simul. 7 (4) (2006) 393-398.

[5] L. Valdevit, S. W. Godfrey, T. A. Schaedler, A. J. Jacobsen, W. B. Carter, Compressive strength of hollow microlattices: Experimental characterization, modeling, and optimal design, J. Mater. Res. 28 (17) (2013) 2461-2473.

[6] N. Wicks, J. W. Hutchinson, Optimal truss plates, Int. J. Solids Struct. 38 (30-31) (2001) 5165-5183. doi:10.1016/S0020-7683(00)00315-2.

[7] S. Engelbrecht, L. Folgar, D. W. Rosen, G. Schulberger, J. Williams, Cellular structures for optimal performance, Proc. SFF Symp. Austin (2009) 831-842. 
[8] A. Ben-Tal, M. P. Bendsoe, A new method for optimal truss topology design, SIAM J. Optim. 3 (2) (1993) 322-358.

[9] W. S. Dorn, R. E. Gomory, H. J. Greenberg, Automatic design of optimal structures, J. Mec. 3 (1964) $25-52$.

[10] M. P. Bendsoe, a. Ben-Tal, J. Zowe, Optimization methods for truss geometry and topology design , Struct. Multidiscip. Optim. 7 (3) (1994) 141-159. doi:10.1007/bf01742459.

[11] G. I. N. Rozvany, M. Zhou, T. Birker, Generalized shape optimization without homogenization, Struct. Optim. 4 (3-4) (1992) 250-252. doi:10.1007/BF01742754.

[12] N. Olhoff, M. P. Bendsoe, J. Rasmussen, On CAD-integrated structural topology and design optimization, Comput. Methods Appl. Mech. Eng. 89 (1-3) (1991) 259-279. doi:10.1016/0045-7825(91)90044-7.

[13] M. P. Bendsoe, N. Kikuchi, Generating Optimal Topologies in Structural Design Using a Homogenization Method, Comput. Methods Appl. Mech. Eng. 71 (2) (1988) 197-224.

URL <Go to ISI>://WOS:A1988R098500006

[14] G. Allaire, F. Jouve, H. Maillot, Topology optimization for minimum stress design with the homogenization method, Struct. Multidiscip. Optim. 28 (2-3) (2004) 87-98. doi:10.1007/s00158-004-0442-8.

[15] G. Allaire, Shape optimization by the homogenization method, Springer-Verlag, New York, 2002.

[16] B. Niu, J. Yan, G. Cheng, Optimum structure with homogeneous optimum cellular material for maximum fundamental frequency, Struct. Multidiscip. Optim. 39 (2) (2009) 115-132. doi:10.1007/s00158008-0334-4.

[17] S. Hyun, S. Torquato, Optimal and manufacturable two-dimensional, Kagome-like cellular solids, J. Mater. Res. 17 (1) (2002) 137-144. doi:10.1557/JMR.2002.0021.

URL papers3://publication/uuid/E534224A-E4C9-4A45-B82F-785908C6AE69

[18] C. Le, T. E. Bruns, D. A. Tortorelli, Material microstructure optimization for linear elastodynamic energy wave management, J. Mech. Phys. Solids 60 (2) (2012) 351-378.

URL http://dx.doi.org/10.1016/j.jmps.2011.09.002

[19] O. Sigmund, Tailoring materials with prescribed elastic properties, Mech. Mater. 20 (4) (1995) 351-368. doi:10.1016/0167-6636(94)00069-7. 
[20] M. M. Neves, O. Sigmund, M. P. Bendsøe, Topology optimization of periodic microstructures with a penalization of highly localized buckling modes, Int. J. Numer. Methods Eng. 54 (6) (2002) 809-834. doi:10.1002/nme.449.

[21] L. Liu, J. Yan, G. Cheng, Optimum structure with homogeneous optimum truss-like material, Comput. Struct. 86 (13-14) (2008) 1417-1425. doi:10.1016/j.compstruc.2007.04.030.

[22] X. Huang, S. W. Zhou, Y. M. Xie, Q. Li, Topology optimization of microstructures of cellular materials and composites for macrostructures, Comput. Mater. Sci. 67 (2013) 397-407. doi:10.1016/j.commatsci.2012.09.018.

[23] A. R. Diaz, A. Benard, Designing materials with prescribed elastic properties using polygonal cells, Int. J. Numer. Methods Eng. 57 (3) (2003) 301-314. doi:10.1002/nme.677.

[24] R. Hutchinson, N. Fleck, The structural performance of the periodic truss, J. Mech. Phys. Solids 54 (4) (2006) 756-782. doi:10.1016/j.jmps.2005.10.008.

URL http://www.sciencedirect.com/science/article/pii/S002250960500205X

[25] A. Srikantha Phani, J. Woodhouse, N. A. Fleck, Wave propagation in two-dimensional periodic lattices, J. Acoust. Soc. Am. 119 (4) (2006) 1995-2005.

[26] M. C. Messner, M. I. Barham, M. Kumar, N. R. Barton, Wave propagation in equivalent continuums representing truss lattice materials, Int. J. Solids Struct. 73-74 (2015) 55-66.

[27] V. Deshpande, N. Fleck, M. Ashby, Effective properties of the octet-truss lattice material, J. Mech. Phys. Solids 49 (8) (2001) 1747-1769. doi:10.1016/S0022-5096(01)00010-2.

URL http://www.sciencedirect.com/science/article/pii/S0022509601000102

[28] L. Gong, S. Kyriakides, N. Triantafyllidis, On the stability of Kelvin cell foams under compressive loads, J. Mech. Phys. Solids 53 (4) (2005) 771-794. doi:10.1016/j.jmps.2004.10.007.

[29] G. Allaire, L. Friz, Localization of high-frequency waves propagating in a locally periodic medium, Proc. R. Soc. Edinburgh 140A (2010) 897-926.

[30] R. V. Craster, J. Kaplunov, a. V. Pichugin, High-frequency homogenization for periodic media, Proc. R. Soc. A Math. Phys. Eng. Sci. 466 (2120) (2010) 2341-2362. doi:10.1098/rspa.2009.0612.

[31] E. Nolde, R. V. Craster, J. Kaplunov, High frequency homogenization for structural mechanics, J. Mech. Phys. Solids 59 (3) (2011) 651-671. doi:10.1016/j.jmps.2010.12.004.

URL http://dx.doi.org/10.1016/j.jmps.2010.12.004 
[32] H. N. G. Wadley, N. a. Fleck, A. G. Evans, Fabrication and structural performance of periodic cellular metal sandwich structures, Compos. Sci. Technol. 63 (16) (2003) 2331-2343. doi:10.1016/S02663538(03)00266-5.

[33] American Institute of Steel Construction, Steel Construction Manual, 14th Edition, 2011.

[34] A. C. Eringen, D. Edelen, On nonlocal elasticity, Int. J. Eng. Sci. 10 (1972) 233-248.

[35] A. C. Eringen, Nonlocal polar elastic continua, Int. J. Eng. Sci. 10 (1) (1972) 1-16. doi:10.1016/0020$7225(72) 90070-5$.

[36] A. C. Eringen, Linear theory of nonlocal elasticity and dispersion of plane waves, Int. J. Eng. Sci. 10 (1972) 425-435. arXiv:83/094703-08\$02.40, doi:10.1016/0020-7225(72)90070-5.

[37] A. C. Eringen, On differential equations of nonlocal elasticity and solutions of screw dislocation and surface waves, J. Appl. Phys. 54 (9) (1983) 4703-4710. arXiv:83/094703-08\$02.40, doi:10.1063/1.332803.

[38] D. Kraft, A software package for sequential quadratic programming, Tech. rep., DLR German Aerospace Center - Institute for Flight Mechanics, Koln, Germany (1988).

[39] E. Jones, T. Oliphant, P. Peterson, A. Others, SciPy: open source scientific tools for Python (2001). URL http://www.scipy.org/

[40] S. I. Ranganathan, M. Ostoja-Starzewski, Universal elastic anisotropy index, Phys. Rev. Lett. 101 (5) (2008) 3-6. doi:10.1103/PhysRevLett.101.055504.

[41] Z. Hashin, S. Shtrikman, A variational approach to the theory of the elastic behaviour of multiphase materials, J. Mech. Phys. Solids 11 (2) (1963) 127-140. doi:10.1016/0022-5096(63)90060-7.

[42] M. S. Elsayed, D. Pasini, Multiscale structural design of columns made of regular octet-truss lattice material, Int. J. Solids Struct. 47 (14-15) (2010) 1764-1774. doi:10.1016/j.ijsolstr.2010.03.003.

URL http://www.sciencedirect.com/science/article/pii/S0020768310000788 http://linkinghub.elsevier.com/retrieve/pii/S0020768310000788

[43] A. Srikantha Phani, N. A. Fleck, Elastic Boundary Layers in Two-Dimensional Isotropic Lattices, J. Appl. Mech. 75 (2008) 021020-1-8. doi:10.1115/1.2775503.

[44] C. V. Camp, B. J. Bichon, S. P. Stovall, Design of Steel Frames Using Ant Colony Optimization 131 (3) (2005) 369-379. 
[45] L. F. F. Miguel, L. F. F. Miguel, Shape and size optimization of truss structures considering dynamic constraints through modern metaheuristic algorithms, Expert Syst. Appl. 39 (10) (2012) 9458-9467. doi:10.1016/j.eswa.2012.02.113.

[46] P. Nakshatrala, D. Tortorelli, Topology optimization for effective energy propagation in rateindependent elastoplastic material systems, Comput. Methods Appl. Mech. Eng. 295 (2015) 305-326. doi:10.1016/j.cma.2015.05.004.

\section{A. Frame element}

The linear frame element used in this work is the combination of linear axial deformation, Euler-Bernoulli bending in two transverse directions, and linear torsion. The element uses:

$$
\mathbf{u}=\left[\begin{array}{llllllllllll}
d_{x}^{(1)} & d_{y}^{(1)} & d_{z}^{(1)} & d_{x}^{(2)} & d_{y}^{(2)} & d_{z}^{(2)} & \theta_{x}^{(1)} & \theta_{y}^{(1)} & \theta_{z}^{(1)} & \theta_{x}^{(2)} & \theta_{y}^{(2)} & \theta_{z}^{(2)}
\end{array}\right]
$$

as the degree-of-freedom vector; with $d$ nodal translations in three directions and $\theta$ nodal rotations about the three axes; and

$$
\mathbf{f}=\left[\begin{array}{llllllllllll}
f_{x}^{(1)} & f_{y}^{(1)} & f_{z}^{(1)} & f_{x}^{(2)} & f_{y}^{(2)} & f_{z}^{(2)} & m_{x}^{(1)} & m_{y}^{(1)} & m_{z}^{(1)} & m_{x}^{(2)} & m_{y}^{(2)} & m_{z}^{(2)}
\end{array}\right]
$$

as the generalized force vector; with $f$ nodal forces and $m$ nodal moments. The superscripts (1) and (2) indicate the left and right nodes of the reference element.

Given this convention, the element stiffness matrix separates into uncoupled blocks representing each deformation mechanism. The axial block:

$$
\left[\begin{array}{l}
f_{x}^{(1)} \\
f_{x}^{(2)}
\end{array}\right]=\frac{A E}{L}\left[\begin{array}{cc}
1 & -1 \\
-1 & 1
\end{array}\right]\left[\begin{array}{l}
d_{x}^{(1)} \\
d_{x}^{(2)}
\end{array}\right]
$$

the two bending blocks:

$$
\left[\begin{array}{c}
f_{y}^{(1)} \\
m_{z}^{(1)} \\
f_{y}^{(2)} \\
m_{z}^{(2)}
\end{array}\right]=\frac{E I_{z z}}{L^{3}}\left[\begin{array}{cccc}
12 & 6 L & -12 & 6 L \\
6 L & 4 L^{2} & -6 L & 2 L^{2} \\
-12 & -6 L & 12 & -6 L \\
6 L & 2 L^{2} & -6 L & 4 L^{2}
\end{array}\right]\left[\begin{array}{c}
d_{y}^{(1)} \\
\theta_{z}^{(1)} \\
d_{y}^{(2)} \\
\theta_{z}^{(2)}
\end{array}\right]
$$


and

$$
\left[\begin{array}{c}
f_{z}^{(1)} \\
m_{y}^{(1)} \\
f_{z}^{(2)} \\
m_{y}^{(2)}
\end{array}\right]=\frac{E I_{y y}}{L^{3}}\left[\begin{array}{cccc}
12 & -6 L & -12 & -6 L \\
-6 L & 4 L^{2} & 6 L & 2 L^{2} \\
-12 & 6 L & 12 & 6 L \\
-6 L & 2 L^{2} & 6 L & 4 L^{2}
\end{array}\right]\left[\begin{array}{c}
d_{z}^{(1)} \\
\theta_{y}^{(1)} \\
d_{z}^{(2)} \\
\theta_{y}^{(2)}
\end{array}\right]
$$

and the torsion block:

$$
\left[\begin{array}{l}
m_{x}^{(1)} \\
m_{x}^{(1)}
\end{array}\right]=\frac{G J}{L}\left[\begin{array}{cc}
1 & -1 \\
-1 & 1
\end{array}\right]\left[\begin{array}{l}
\theta_{x}^{(1)} \\
\theta_{x}^{(2)}
\end{array}\right]
$$

The mass matrix can be similarly divided into an axial block

$$
\left[\begin{array}{l}
f_{x}^{(1)} \\
f_{x}^{(2)}
\end{array}\right]=\frac{\rho A L}{6}\left[\begin{array}{ll}
2 & 1 \\
1 & 2
\end{array}\right]\left[\begin{array}{l}
\ddot{d}_{x}^{(1)} \\
\ddot{d}_{x}^{(2)}
\end{array}\right]
$$

the two bending blocks

$$
\begin{gathered}
{\left[\begin{array}{c}
f_{y}^{(1)} \\
m_{z}^{(1)} \\
f_{y}^{(2)} \\
m_{z}^{(2)}
\end{array}\right]=\frac{\rho A L}{420}\left[\begin{array}{cccc}
156 & 22 L & 54 & -13 L \\
22 L & 4 L^{2} & 13 L & -3 L^{2} \\
54 & 13 L & 156 & -22 L \\
-13 L & -3 L^{2} & -22 L & 4 L^{2}
\end{array}\right]\left[\begin{array}{l}
\ddot{d}_{y}^{(1)} \\
\ddot{\theta}_{z}^{(1)} \\
\ddot{d}_{y}^{(2)} \\
\ddot{\theta}_{z}^{(2)}
\end{array}\right]} \\
{\left[\begin{array}{c}
f_{z}^{(1)} \\
m_{y}^{(1)} \\
f_{z}^{(2)} \\
m_{y}^{(2)}
\end{array}\right]=\frac{\rho A L}{420}\left[\begin{array}{cccc}
156 & -22 L & 54 & 13 L \\
-22 L & 4 L^{2} & -13 L & -3 L^{2} \\
54 & -13 L & 156 & 22 L \\
13 L & -3 L^{2} & 22 L & 4 L^{2}
\end{array}\right]\left[\begin{array}{l}
\ddot{d}_{z}^{(1)} \\
\ddot{\theta}_{y}^{(1)} \\
\ddot{d}_{z}^{(2)} \\
\ddot{\theta}_{y}^{(2)}
\end{array}\right]}
\end{gathered}
$$

and the torsion block

$$
\left[\begin{array}{l}
m_{x}^{(1)} \\
m_{x}^{(1)}
\end{array}\right]=\frac{\rho J L}{6}\left[\begin{array}{ll}
2 & 1 \\
1 & 2
\end{array}\right]\left[\begin{array}{l}
\ddot{\theta}_{x}^{(1)} \\
\ddot{\theta}_{x}^{(2)}
\end{array}\right] .
$$

In these expressions $A$ is the strut cross-sectional area, $E$ the bulk material Young's modulus, $L$ the strut length, $I_{z z}$ the section moment of inertia about the $z$-axis, $I_{y y}$ the section moment of inertia about the $y$-axis, $G$ the bulk material shear modulus, $J$ the section torsion constant, and $\rho$ the bulk material density. For solid circular cross-sections all the geometric quantities are functions of the strut cross-sectional area:

$$
I_{z z}=I_{y y}=\frac{A^{2}}{4 \pi}
$$


and

$$
J=\frac{A^{2}}{2 \pi} .
$$

\section{Vitae}

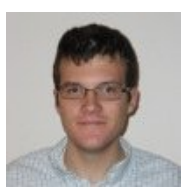

Mark Messner is currently a post-doctoral researcher at Lawrence Livermore National Laboratory, having graduated with a $\mathrm{PhD}$ from the University of Illinois at Urbana-Champaign. 